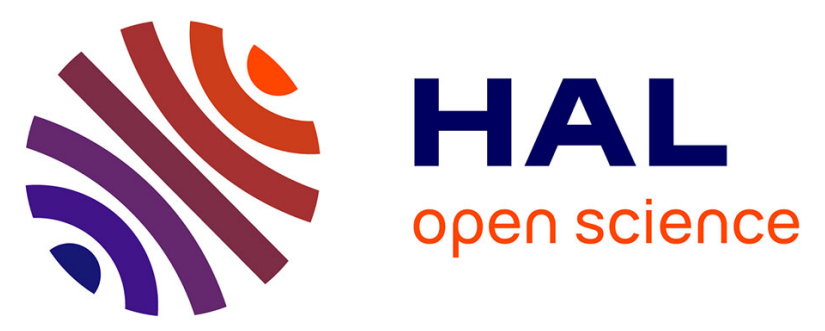

\title{
Substrate specificity and inhibitors of LRRK2, a protein kinase mutated in Parkinson's disease
}

R. Jeremy Nichols, Nicolas Dzamko, Jessica E. Hutti, Lewis C. Cantley, Maria Deak, Jennifer Moran, Paul Bamborough, Alastair D. Reith, Dario R. Alessi

\section{- To cite this version:}

R. Jeremy Nichols, Nicolas Dzamko, Jessica E. Hutti, Lewis C. Cantley, Maria Deak, et al.. Substrate specificity and inhibitors of LRRK2, a protein kinase mutated in Parkinson's disease. Biochemical Journal, 2009, 424 (1), pp.47-60. 10.1042/BJ20091035 . hal-00479223

\section{HAL Id: hal-00479223 \\ https://hal.science/hal-00479223}

Submitted on 30 Apr 2010

HAL is a multi-disciplinary open access archive for the deposit and dissemination of scientific research documents, whether they are published or not. The documents may come from teaching and research institutions in France or abroad, or from public or private research centers.
L'archive ouverte pluridisciplinaire HAL, est destinée au dépôt et à la diffusion de documents scientifiques de niveau recherche, publiés ou non, émanant des établissements d'enseignement et de recherche français ou étrangers, des laboratoires publics ou privés. 


\section{Substrate specificity and inhibitors of LRRK2, a protein kinase mutated in Parkinson's disease.}

Ry. Jeremy Nichols ${ }^{1}$, Nicolas Dzamko ${ }^{1}$, Jessica E. Hutti ${ }^{2}$, Lewis C Cantley ${ }^{2,}$ Maria Deak $^{1}$, Jennifer Moran ${ }^{3}$, Paul Bamborough ${ }^{5}$, Alastair D Reith ${ }^{4}$ and Dario R. Alessi ${ }^{1}$

1. MRC Protein Phosphorylation Unit, College of Life Sciences, University of Dundee, Dow Street, Dundee DD1 5EH, Scotland.

2. Department of Systems Biology, Harvard Medical School, Boston, Massachusetts and Division of Signal Transduction, Beth Israel Deaconess Medical Center, Boston,

Massachusetts.

3. Division of Signal Transduction Therapy Unit, College of Life Sciences, University of Dundee, Dow Street, Dundee DD1 5EH, Scotland.

4. Neurosciences Centre of Excellence for Drug Discovery, GlaxoSmithKline

Pharmaceuticals R\&D, New Frontiers Science Park, Third Avenue, Harlow, Essex CM19 5AW, UK.

5 Molecular Discovery Research, GlaxoSmithKline Pharmaceuticals R\&D, Medicines

Research Centre, Gunnels Wood Road, Stevenage, UK

Correspondence to RJN (r.j.nichols@dundee.ac.uk)

Tel +44 1382385602

Fax +44 1382223778

Short Title: Substrate Specificity and Inhibitors of LRRK2 


\begin{abstract}
The Leucine Rich Repeat Protein Kinase-2 (LRRK2) is mutated in a significant number of Parkinson's disease patients, but little is known about its regulation and function. A common mutation changing Gly2019 to Ser enhances catalytic activity, suggesting small molecule inhibitors might have utility in treating Parkinson's Disease. We utilised various approaches to explore the substrate specificity requirements of LRRK2 and elaborated a peptide substrate termed Nictide, that had 20 -fold lower $\mathrm{K}_{\mathrm{m}}$ and nearly 2 -fold higher $\mathrm{V}_{\max }$ than the widely deployed LRRKtide substrate. We demonstrate that LRRK2 has marked preference for phosphorylating Thr over Ser. We also observed that several Rho kinase (ROCK) inhibitors such as Y-27632 and H-1152, suppressed LRRK2 with similar potency to which they inhibited ROCK2. In contrast, GSK429286A a selective ROCK inhibitor, did not significantly inhibit LRRK2. We also identified a mutant LRRK2[A2016T] that was normally active, but resistant to H-1152, Y-27632 as well as sunitinib, a structurally unrelated multikinase inhibitor that in contrast to other compounds suppresses LRRK2, but not ROCK. We have also developed the first sensitive antibody that enables measurement of endogenous LRRK2 protein levels and kinase activity as well as shRNA methods to reduce LRRK2 expression. Finally, we describe a pharmacological approach to validate whether substrates are phosphorylated by LRRK2 and use this to provide evidence that LRRK2 may not be rate-limiting for the phosphorylation of the proposed substrate moesin. Our findings reported in this study will aid with the investigation of the LRRK2 kinase.
\end{abstract}




\section{Introduction}

Autosomal dominant point mutations within the gene encoding for the Leucine Rich Repeat protein Kinase-2 (LRRK2) predispose humans to Parkinson's disease (PD) [1, 2]. Patients with LRRK2 mutations generally develop PD at the normal age of 60-70 years, with clinical appearance and symptoms indistinguishable from idiopathic PD [3]. Mutations in LRRK2 account for $4 \%$ of familial PD, and observed in $1 \%$ of sporadic PD patients [3]. Little is known about how LRRK2 is regulated, what are its substrates and how mutations cause PD.

LRRK2 is a large multi-domain protein kinase of 2527 residues, consisting of leucine rich repeats (residues 1010-1287), GTPase domain (residues 1335-1504), COR domain (residues 1517-1843), serine/threonine protein kinase domain (residues 1875-2132) and a WD40 repeat (residues 2231-2276) [4]. Over 40 mutations have thus far been reported which mainly comprise amino acid substitutions [5]. The most frequent mutation comprises an amino acid substitution of the highly conserved Gly2019 located within the subdomain VII-DFG motif of the kinase domain to a Ser residue [5]. Several studies have reported that this mutation enhances the protein kinase activity of LRRK2 two to three-fold, suggesting that LRRK2 inhibitors may have utility for the treatment of PD [6]. Other than non-specific/multi-kinase protein kinase inhibitors staurosporine $\left(\mathrm{IC}_{50} 2 \mathrm{nM}\right), \mathrm{K} 252\left(\mathrm{IC}_{50} 4 \mathrm{nM}\right)$, Su-11248/sunitinib $\left(\mathrm{IC}_{50} 15 \mathrm{nM}\right)[7,8]$ no selective LRRK2 inhibitors have been reported thus far.

We previously undertook a KESTREL screen in rat brain extracts to identify proteins phosphorylated by the activated PD LRRK2[G2019S] mutant. This led to the observation that moesin, a member of the ERM proteins that anchors the actin-cytoskeleton to the plasma membrane is efficiently phosphorylated by LRRK2, at Thr558, a previously identified in vivo phosphorylation site that regulates the ability of moesin to bind actin [9]. LRRK2 also phosphorylated other ERM proteins, ezrin and radixin that are related to moesin, at the residue equivalent to Thr558, as well as a peptide encompassing Thr558 (LRRKtide) [9]. Previous work had suggested that the Rho-kinase (ROCK) could also phosphorylate ERM proteins at the residue equivalent to Thr558 of moesin both in vitro and when overexpressed in cells [10-12]. No evidence has been published to demonstrate that LRRK2 phosphorylates ERM proteins in cells.

To aid the functional characterisation of LRRK2, we have analysed the substrate specificities of LRRK2 and elaborated the peptide substrate Nictide that has a 20-fold lower $\mathrm{K}_{\mathrm{m}}$ and nearly 2-fold higher $\mathrm{V}_{\max }$ than the widely deployed LRRKtide substrate [9]. We also observed that some previously reported ROCK inhibitors also inhibited LRRK2 with similar potency as they inhibited ROCK. Moreover, we demonstrate that sunitinib can be deployed as a control compound that inhibits LRRK2 but not ROCK and the specific ROCK inhibitor GSK429286A [13] can be deployed as a compound that inhibits ROCK, but not LRRK2. Modelling studies enabled us to generate an inhibitor-resistant mutant of LRRK2 that is normally active, but 20 -fold less sensitive to inhibition by the LRRK2 inhibitors. We also develop the first robust assay that allows the protein kinase activity of endogenous LRRK2 to be quantified and present a pharmacological strategy that can be deployed to validate LRRK2 substrates. The findings presented in this study will help with dissecting the regulation and function of LRRK2. 


\section{Materials and Methods}

Antibodies. A glutathione-s transferase (GST) fusion protein of amino acids was expressed in bacteria and purified by glutathione-Sepharose chromatography. Following cleavage of the GST tag, LRRK2(residues 100-500) was used as an immunogen to raise a polyclonal antibody (S348C). Antibodies were affinity purified from antisera using the LRRK2(residues 100-500) protein immunogen. Antibody (S374C) against LRRK2 was raised against a peptide immunogen similar to what was described previously [14], encompassing amino acids 2498-2514 (CINLPHEVQNLEKHIEVR with NH2 cysteine for coupling to keyhole limpet hemocyanin[KLH]). Antibodies were affinity purified against the peptide. Antimoesin (S135C) and anti-ezrin antibodies (S245C) were generated by injection of purified full-length protein into sheep, followed by affinity purification of the antibody against the antigen. Pan phospho-ERM antibody (S296C) was generated by injection of the KLH conjugated phosphopeptide CDKYKTpLRQI into sheep and was affinity purified by positive and negative selection against the phospho and de-phospho peptides respectively. Sheep polyclonal antibody (S662B) was raised against MBP-MYPT chicken amino acids (7141004). Rabbit polyclonal antibody against MYPT phosphothreonine 850 was from Upstate (\# 36-003). Anti GFP antibody (S268B) was raised against recombinant GFP protein and affinity purified against the antigen. Antibody (S221B) against ERK1/2 was raised against GST-ERK1 protein. Anti-FLAG M2 antibody and affinity matrix were from Sigma (A2220).

LRRK2 and ROCK kinase assays. Peptide Kinase Assays were set up in a total volume of $40 \mu \mathrm{l}$ with either recombinant GST-LRRK2(residues 1326-2527) $(0.5 \mu \mathrm{g}$, which is $\sim 10 \%$ pure [9] thus corresponding to $8 \mathrm{nM}$ LRRK2 in final kinase assay) or immunoprecipitated LRRK2 or recombinant His-ROCK2(residues 2-543) (80 ng, corresponding to $30 \mathrm{nM}$ ROCK2 in final kinase assay) in 50mM Tris $\mathrm{pH} 7.5,0.1 \mathrm{mM}$ EGTA, $10 \mathrm{mM} \mathrm{MgCl}_{2}$ and $0.1 \mathrm{mM}\left[\gamma^{-}{ }^{32} \mathrm{P}\right] \mathrm{ATP}$ $(\sim 500-1000 \mathrm{cpm} / \mathrm{pmol})$ in the presence of the indicated concentration of peptide substrate. In reactions where kinase inhibitors were assayed, inhibitors were dissolved in dimethyl sulfoxide and were at $0.1 \%$ of the reaction volume. After incubation for $15 \mathrm{~min}$ at $30^{\circ} \mathrm{C}$, reactions were terminated by applying $35 \mu 1$ of the reaction mixture on to P81

phosphocellulose paper and immersion in $50 \mathrm{mM}$ phosphoric acid. After extensive washing, reaction products were quantitated by Cerenkov counting. A unit (U) of LRRK2 activity was defined as the amount of enzyme that catalysed the incorporation of $1 \mathrm{nmol}$ of ${ }^{32} \mathrm{P}$ into LRRKtide. $K_{\mathrm{m}}$ and $V_{\max }$ parameters were determined by performing the assay described above using various concentrations of LRRKtide or Nictide. The $K_{\mathrm{m}}$ and $V_{\max }$ parameters were calculated using the Graph-Pad Prism program. $K_{\mathrm{m}}$ and $V_{\max }$ values are rounded in Figure $1 \mathrm{C}$ to reflect the need to estimate values due to the nature of the peptide assay, wherein high concentrations of peptide became inhibitory to the kinase. IC50 values were calculated using non-linear regression analysis using Graph-Pad Prism.

For assays using recombinant proteins as substrates, the assays were set up in a total volume of $25 \mu 1$ with recombinant kinase (12 nM-GST-LRRK2(residues 1326-2527) or $5 \mathrm{nM}$ HisROCK2(residues 2-543)) in 50mM Tris pH 7.5, 0.1mM EGTA, $10 \mathrm{mM} \mathrm{MgCl}$ and $0.1 \mathrm{mM}$ $\left[\gamma-{ }^{32} \mathrm{P}\right]$ ATP $(\sim 500 \mathrm{cpm} / \mathrm{pmol})$, with substrate at $2 \mu \mathrm{M}$. After incubation for $15 \mathrm{~min}$ at $30^{\circ} \mathrm{C}$, the reactions were stopped by the addition of Laemelli sample buffer. Reaction products were resolved by electrophoresis on sodium dodecyl sulfate polyacrylamide gels. The

incorporation of phosphate into protein substrates was determined by autoradiography and/or immunoblotting with phosphospecific antibodies.

Immunological procedures. Cell lysates (10-30ug) were resolved by electrophoresis on SDS polyacrylamide gels or Novex $4-12 \%$ gradient gels, and electroblotted to nitrocellulose membranes. Membranes were blocked with $5 \%$ milk (w/v) in $50 \mathrm{mM}$ Tris/HCl, pH 7.5, 0.15 $\mathrm{M} \mathrm{NaCl}$ and $0.1 \%(\mathrm{v} / \mathrm{v})$ Tween (TBST Buffer). For phospho-MYPT antibody, primary antibody was used at a concentration of $1 \mu \mathrm{g} / \mathrm{ml}$, diluted in 5\% BSA in TBST. Phospho-ERM antibody was used at $1 \mu \mathrm{g} / \mathrm{ml}$ in the presence of $10 \mu \mathrm{g} / \mathrm{ml}$ LRRKtide, diluted in $5 \%(\mathrm{w} / \mathrm{v})$ skimmed milk in TBST. All other antibodies were used at $1 \mu \mathrm{g} / \mathrm{ml}$ in $5 \%(\mathrm{w} / \mathrm{v})$ milk in TBST. Detection of immune-complexes was performed using horseradish-peroxidase- 
conjugated secondary antibodies (Pierce) and an enhanced-chemiluminescence reagent. For immunoprecipitations, antibody was non-covalently coupled to protein G-Sepharose at a ratio of $1 \mu \mathrm{g}$ antibody/ $\mu \mathrm{l}$ of beads, or anti-FLAG M2-agarose was utilized. The indicated amount of cell lysate was incubated with $5 \mu \mathrm{l}$ bed volume of coupled antibody for 1 hour. Immune complexes were washed twice with lysis buffer supplemented with $0.5 \mathrm{M} \mathrm{NaCl}$ and twice with Buffer A. Precipitates were either used as a source of kinase or immediately analyzed by immunoblot.

Lentivirus shRNA production and transduction. For knockdown of endogenous mouse LRRK2, MISSION $^{\mathrm{TM}}$ shRNA constructs in the lentiviral expression vector pLKO.1-Puro were purchased from Sigma. Clones TRCN0000022655 termed shRNA LRRK2 \#1 (CCGGCGCAGCTTTCAGCGATTCTAACTCGAGTTAGAATCGCTGAAAGCTGCGTT TTT) and TRNC0000022654 termed shRNA LRRK2 \#2 (CCGGGCTTACTACTTCACGATATTTCTCGAGAAATATCGTGAAGTAGTAAGCTTT TT) were used for experiments. A non-targeting scrambled shRNA construct (CCGGCCTAAGGTTAAGTCGCCCTCGCTCTAGCGAGGGCGACTTAACCTTAGGTT TTT) also in pLKO.1-Puro was used as a control. For the production of lentiviral transduction particles, HEK293T cells growing in $10 \mathrm{~cm}$ dishes were transfected with $11 \mu \mathrm{g}$ shRNA encoding plasmid along with $7 \mu \mathrm{g}$ of the packaging plasmid pCMV delta R8.2 and 4 $\mu \mathrm{g}$ of the envelope plasmid pCMV-VSV-G using the polyethylamine method. At 24 and $48 \mathrm{~h}$ post-transfection virus containing media was collected. Media from both time points was pooled, centrifuged at $1500 \mathrm{rpm}$ for $5 \mathrm{~min}$, filtered through a $0.2 \mu \mathrm{m}$ filter, aliquoted and stored at $-80^{\circ} \mathrm{C}$. For lentiviral transduction of RAW 264.7 cells (Mouse leukaemic monocyte macrophage cell line), $1 \mathrm{ml}$ of viral supernatant and $5 \mu \mathrm{g} / \mathrm{ml}$ polybrene was added to cells at $50 \%$ confluence in 6 well dishes. Virus containing media was removed after $24 \mathrm{~h}$ and successfully infected cells were selected with the addition of $5 \mu \mathrm{g} / \mathrm{ml}$ puromycin to the media. Cells were maintained in media with $5 \mu \mathrm{g} / \mathrm{ml}$ puromycin until being harvested for experiments.

The Supplementary methods section contains further detailed information on the materials, general methods, buffers, cell culture, purification of recombinant proteins, the specificity kinase panel, mass spectrometry methodology, computer analysis and alternative procedure to verify binding of phosphorylated peptides to P81 paper that are employed in this study 


\section{Results}

Comparison of the substrate specificities of LRRK2 and ROCK. We first compared the rates at which recombinant ROCK2 (residues 2-543) and a fragment of LRRK2 (residues 1326-2527) lacking the N-terminal non-catalytic leucine rich repeats, phosphorylated ezrin and MYPT (a well characterised ROCK substrate [15]). Under conditions in which equimolar MYPT and ezrin were present, ROCK2 phosphorylated MYPT but barely ezrin (Fig 1A). In contrast, LRRK2 phosphorylated ezrin, but did not phosphorylate MYPT (Fig 1A). Comparison of residues surrounding Thr850 (major ROCK phosphorylation site on MYPT [16]) and Thr567 (LRRK2 phosphorylation site on ezrin [9]), revealed that overall these peptides were dissimilar with only the positions $-3\left(\mathrm{R}_{\mathrm{MYPT}} / \mathrm{K}_{\mathrm{ezrin}}\right)$ possessing homology (Fig 1A, lower panel).

The human sequence surrounding the LRRK2 phosphorylation site of ezrin is identical in moesin and radixin, and also strikingly conserved in Drosophila and C.elegans ERM homologues (Fig 1B, lower panel). To investigate the substrate specificity determinants of LRRK2, we tested how mutation of different residues affected the kinetics of LRRK2 phosphorylation of the LRRKtide peptide that encompasses the Thr567 ERM phosphorylation motif (Fig 1C). The wild type LRRKtide peptide was phosphorylated by LRRK2 with a $\mathrm{K}_{\mathrm{m}}$ of $200 \mu \mathrm{M}$ and $\mathrm{V}_{\max }$ of $14 \mathrm{U} / \mathrm{mg}$. The following mutations to Ala suppressed phosphorylation by increasing the $K_{m}$ value: -5Arg ( 2.5 fold), $-2 \operatorname{Tyr}$ (2.4-fold), $+2 \operatorname{Arg}$ (4.5-fold) and +5Arg (4-fold). Mutation of the -2Tyr to Glu increased $\mathrm{K}_{\mathrm{m}} 4.4$-fold, whilst its mutation to Arg slightly decreased $K_{m}$ suggesting that an aromatic residue at this position is not essential (Fig 1C). Mutation of the +2 and +5 residues of the peptide to Pro or Glu increased $\mathrm{K}_{\mathrm{m}}$ similarly to the Ala mutation. Only mutation of the -4 (Asp) to Ala moderately enhanced peptide phosphorylation by decreasing $K_{m} 2.3$-fold (Fig 1C). Several mutations also markedly decreased $\mathrm{V}_{\max }$ values which included: -2 Tyr (2 to 10-fold), +1 Leu (2-fold), +2 Arg (4 to 28 -fold) and +5 Arg (mutation to Glu 4-fold). Binding of phosphopeptides to P81 paper was used as our assay to isolate and quantify phosphorylation of peptides (see Materials and Methods). In order to verify that substitution of residues in LRRKtide did not affect binding to P81 paper, 7 different phosphorylated peptides were purified by HPLC and demonstrated that to interact with similarly high efficiency to P81 paper (Supp Fig 1).

We also investigated how mutations in LRRKtide affected phosphorylation by ROCK2. In contrast to LRRK2, we observed that several mutations substantially improved peptide phosphorylation by decreasing the $\mathrm{K}_{\mathrm{m}}$ value. The most dramatic change involved mutation of the -2Tyr to an Arg residue that is found in most ROCK substrates. This decreased the $\mathrm{K}_{\mathrm{m}}$ value over 60 -fold and increased the $V_{\max }$ over 5 -fold. Mutation of the +1 Leu residue to Ala abolished phosphorylation by ROCK2, but had no effect on LRRK2 phosphorylation (Fig 1C).

Elaboration of an optimal LRRK2 peptide substrate. To further investigate and improve the optimal phosphorylation motif for LRRK2, we utilized a positional scanning peptide library approach [17, 18]. This assay utilises 198 biotinylated peptide libraries. Each library contains a 1:1 mixture of serine and threonine at the central position and one additional position fixed to one of the 20 amino acids, phosphothreonine, or phosphotyrosine. Phosphothreonine and phosphotyrosine were included to allow identification of kinases that possess a requirement for priming phosphorylation events. All other positions contain an equimolar degenerate mixture of natural amino acids (except serine, threonine, and cysteine). Recombinant LRRK2[G2019S] or kinase inactive LRRK2[D2017A] was used to phosphorylate all 198 peptide libraries simultaneously in solution using $\gamma^{32} \mathrm{P}-\mathrm{ATP}$, and biotinylated peptides were captured on a streptavidin-coated membrane. The relative preference for each amino acid at each position was determined by quantifying ${ }^{32} \mathrm{P}$ radioactivity incorporation following phosphoimaging (Fig $2 \mathrm{~A}$ ). The quantitative results of the LRRK2[G2019S] screen were also input as a matrix into enoLOGOS programme [19] and the relative preferences for each amino acid is displayed in Figure 2B. We found that LRRK2 exhibited preferred sequence specificity at multiple positions relative to the phosphorylation site, with strong preferences for -5(Trp, Arg), -2(Phe, Tyr, His and Thr), - 
1(Tyr, Arg, Trp), +2 (Arg and Thr) and +3 (Arg) positions. This is consistent with the kinetic studies shown in Figure 1, demonstrating that mutation of these residues increased $\mathrm{K}_{\mathrm{m}}$ and in some cases also decreased $V_{\max }$ values. An Asp or Glu residue at any position with the peptide reduced LRRK2 phosphorylation (Fig 2A). For experiments undertaken with kinaseinactive LRRK2[D2017A], vastly lower overall levels of phosphorylation were observed, but nevertheless some contaminant -kinase activity with preference for Arg residues at the -3 and -2 positions was still found. Similar results were also reported in previous studies employing recombinant kinase-inactive GST-IкB Kinase- $\beta$ derived from 293 cells [20]. This trace level of protein kinase activity likely results from protein kinases that contaminate the GSTpurified kinase from 293 cell extracts.

\section{Elaboration of Nictide LRRK2 substrate.}

The data from the positional scanning peptide library indicated that the optimal LRRK2 phosphorylation motif between -5 and +4 positions is WWRFYTLRRA. In order to generate an improved substrate for LRRK2, we substituted this motif into the moesin sequence, from which the LRRK2tide peptide was derived. Since sequences as distant as the +5 residues affected kinetics of LRRKtide phosphorylation (Fig 1C) and the LRRKtide peptide terminated at the +6 position, we decided to incorporate the WWRFYTLRRA motif into a longer variant of the LRRKtide peptide encompassing a further 6 residues of moesin. The resulting sequence RLGWWRFYTLRRARQGNTKQR was termed Nictide (reflecting the names of the first two authors of this study). We first compared the phosphorylation by LRRK2[G2019S] of GST fused to the original LRRKtide sequence, the longer version of LRRKtide, the entire C-terminus of moesin (residues 500-577) as well as Nictide. This revealed that GST-Nictide was phosphorylated to a significantly greater extent by LRRK2 than the other GST-fusion proteins (Fig 2C). Mutation of the Thr residue predicted to comprise the LRRK2 phosphorylation site, virtually abolished phosphorylation of the GSTfusion proteins. Our results also demonstrate that the expanded LRRKtide sequence was more efficiently phosphorylated by LRRK2 than the original shorter variant (Fig 2C).

We next generated the synthetic Nictide peptide and found that it was phosphorylated by LRRK2[G2019S] with a $K_{m}$ of $10 \mu \mathrm{M}$ (20-fold lower than LRRKtide) and $V_{\max }$ of $26 \mathrm{U} / \mathrm{mg}$ (1.7-fold higher than LRRKtide). Nictide was phosphorylated by wild type LRRK2 with a similar $K_{m}$, but lower $V_{\max }$, consistent with previous work showing that the Gly2019Ser mutation stimulates LRRK2 activity [6]. We also studied the effects of mutating individual residues of Nictide on phosphorylation by LRRK2[G2019S] as well as wild type LRRK2 (Fig 3). The mutations affected wild type LRRK2 and LRRK2[G2019S] similarly. Most mutations moderately affected $\mathrm{K}_{\mathrm{m}}$ values with the largest effect being the $-5 \operatorname{Trp}$ to Ala mutation increasing the $K_{m}$ value 3 -fold. Other mutations decreased the $V_{\max }$ value of phosphorylation 2 to 5-fold (-4Trp to Ala and +2 Arg to Ala) (Fig 3). Several mutations (5Trp to Ala, -4Trp to Ala and -1 Tyr to Ala) increased $\mathrm{K}_{\mathrm{m}}$ values 2 to 3-fold, but enhanced $\mathrm{V}_{\max }$ of LRRK2 phosphorylation $\sim 2$-fold. We also combined the $-5,-4$ and -1 mutations that enhanced $V_{\max }$ and found that although high $V_{\max }$ values were maintained, the $\mathrm{K}_{\mathrm{m}}$ values were substantially increased 5 to 20 -fold compared to Nictide. Interestingly, mutation of the Thr residue phosphorylated by LRRK2 to a Ser, abolished phosphorylation of the peptide by LRRK2 (Fig 3). Surprisingly, mutation of the +1 Leu to a Pro residue, which would inhibit phosphorylation of most substrates by non- CMGC proline directed kinases, only decreased $\mathrm{V}_{\max }$ of LRRK2 phosphorylation under 2-fold without affecting $\mathrm{K}_{\mathrm{m}}$. This suggests that LRRK2 despite not belonging to the CMGC kinase family does have the potential to phosphorylate Ser/Thr residues followed by a Pro residue. The positional scanning peptide library data also indicated that there could be a preference for a Thr residue at the -2 and +2 positions (Fig 2A). As similar preference for Thr at -2 and +2 positions has also been observed in other kinase scanning peptide library screens[21], we decided to introduce Thr at either -2 or +2 positions and found that this moderately increased the $\mathrm{K}_{\mathrm{m}}$ and reduced the $\mathrm{V}_{\max }$ value (Fig 3). Introduction of Thr residues at both the -2 and +2 positions increased $\mathrm{K}_{\mathrm{m}}$ value of over 10-fold suggesting that $\mathrm{Thr}$ at these positions are not well tolerated. To verify that substitution of residues in Nictide did not affect binding to P81 paper we purified 5 different phosphorylated peptides by HPLC and demonstrated that they all interacted with similar high efficiency to P81 paper (Supp Fig 1). 
We also compared how other LRRK2 Parkinson's disease associated mutations phosphorylated Nictide compared to LRRKtide. Using both peptides we found similar results to what we previously reported [9], namely 1.5-2-fold elevated activity for LRRK2[G2019S] and 2-4-fold reduced activity for LRRK2[I2012T], LRRK2[I2020T] and LRRK2[G2385R] (Supp Fig 2).

\section{Identification of selective small molecule tool inhibitors of LRRK2}

In the course of comparing the substrate specificity of LRRK2[G2019S] and ROCK2, we observed that several commonly deployed ROCK inhibitors (Y-27632 [22], hydroxyfasudil [23], H-1152[24]) also inhibited LRRK2[G2019S] (Fig 4A \& B). Y-27632, inhibited LRRK2[G2019S] ( $\left.\mathrm{IC}_{50} 1 \mu \mathrm{M}\right)$ with similar potency to ROCK2 $\left(\mathrm{IC}_{50} 0.7 \mu \mathrm{M}\right)$.

LRRK2[G2019S] was inhibited by hydroxyfasudil $\left(\mathrm{IC}_{50} 6.8 \mu \mathrm{M}\right)$ and $\mathrm{H}-1152\left(\mathrm{IC}_{50} 0.15 \mu \mathrm{M}\right)$ $\sim 3$-fold more weakly than ROCK (Fig 4B). H-1152 and hydroxyfasudil belong to a wellstudied series of isoquinolinesulfonamides [25] whilst Y-27632 is structurally unrelated (Fig 4A). Other isoquinolinesulfonamides reported to inhibit ROCK namely H89 [26] and fasudil [27] only inhibited LRRK2[G2019S] weakly (Fig 4B). In contrast, the dihydropyridone indazole amide ROCK inhibitor GSK 429286A [13] and the aminofurazan ROCK inhibitor GSK269962A [28] were observed to suppress ROCK ( $\left.\mathrm{IC}_{50} \sim 60 \mathrm{nM}\right)$, but not LRRK2 $\left(\mathrm{IC}_{50}>\right.$ $1000 \mathrm{nM}$ ). We confirmed that structurally unrelated sunitinib, inhibited LRRK2 with similar potency $\left(\mathrm{IC}_{50} \sim 19 \mathrm{nM}\right)$ to previous reports $[7,8]$, but in contrast to $\mathrm{Y}-27632$ and $\mathrm{H}-1152$, sunitinib only weakly inhibited ROCK $\left(\mathrm{IC}_{50} \sim 3700 \mathrm{nM}\right)$. Comparing the potency of $\mathrm{H}-1152$, Y-27632 and sunitinib for LRRK2[G2019S] and wild type LRRK2, we observed that wild type LRRK2 was moderately less sensitive to these drugs than the activated mutant. The $\mathrm{IC}_{50}$ of inhibition of wild type LRRK2 was increased 2-fold for H-1152 and Y-27632 and 4-fold for sunitinib (Fig 4B).

\section{LRRK2 tool compound selectivity profiles}

To compare the relative selectivity profiles of Y-27632, H-1152 and fasudil (HA-1077) with GSK429286A, GSK269962A and sunitinib, we profiled these inhibitors against a panel of 85 protein kinases at ATP concentrations, which approximate the $\mathrm{K}_{\mathrm{m}}$ constant for ATP (Table 1). This revealed that GSK429286A is a very selective ROCK2 inhibitor and at $1 \mu \mathrm{M}$ it reduced ROCK 2 activity over 20 -fold, under conditions in which the only other kinase on the panel that was significantly inhibited was MSK1 whose activity was reduced $\sim 5$-fold. Y27632 is also selective, and at $10 \mu \mathrm{M}$ in addition to inhibiting ROCK2 only suppressed activity of PRK2 and MNK1 over 5-fold. H-1152 (1 $\mu \mathrm{M})$ in addition to ROCK, inhibited Aurora B and BRSK2 over 5-fold. Fasudil, hydroxyfasudil, sunitinib and GSK269962A were less selective. Fasudil (10 $\mathrm{MM}$ ) inhibited RSK1, S6K1, PRK2, MSK1, MNK1, MELK, MSK1, MELK over 5-fold. Hydroxyfasudil $(10 \mu \mathrm{M})$ inhibited RSK1, S6K1, PRK2, MSK1. Sunitinib $(1 \mu \mathrm{M})$ and GSK269962A significantly inhibited 12 and 7 kinases respectively out of the 85 kinases profiled (Table 1).

\section{LRRK2 Homology model and development of inhibitor resistant mutants.}

In the absence of an available crystal structure of LRRK2 kinase domain, we utilized the observation that some ROCK1 inhibitors displayed similar activity against LRRK2, to use ROCK1 as a template from which to construct a homology model of the LRRK2 kinase domain. An unpublished structure of ROCK1 complexed with Fasudil, similar to the published structure of ROCK2 [29], was used as the template for the model because of its slightly higher resolution. A binding model of H-1152 in LRRK2 was obtained by superimposing the protein C $\alpha$ atoms of the LRRK2 model with the reported ROCK $1 / \mathrm{H}-1152$ complex [30] (Fig 5A). The binding mode of H-1152 to LRRK2 is predicted to be similar to the binding mode to ROCK1 [30]. In the complex with ROCK1, the isoquinoline nitrogen of $\mathrm{H}-1152$ accepts the hinge hydrogen bond from the backbone NH atom of Met156, and the same interaction is predicted to take place with the backbone $\mathrm{NH}$ atom of the equivalent LRRK2 residue Ala1950. The two methyl groups of H-1152 help to restrict the conformational freedom of the inhibitor, in addition to making lipophilic contacts with the ATP site [30]. These lipophilic contacts are also well conserved in LRRK2. 
The model also highlights the position of Ala2016 in which the side chain $\mathrm{C} \beta$ atom of Ala2016 is located close to H-1152, $4.3 \AA$ away from the homopiperazine methyl group. The $\mathrm{C} 7$ atom of the isoquinoline ring is even closer, only $3.7 \AA$ away. In the case of ROCK2, the residue equivalent to Ala2016 is Ala215 (Fig 5B). This residue in both LRRK2 and ROCK1 lies just prior to the subdomain VII-DFG motif. Previous work has shown that the Ala215 residue on ROCK plays an important role in controlling the specificity of interaction with $\mathrm{H}$ 1152 by forming two van der Waals interactions with H-1152 [30]. PKA is inhibited $~ 50$-fold more weakly by H-1152, and has Thr183 in the equivalent position. Mutation of Thr183 to Ala in PKA did not affect basal activity, but enhanced its inhibition by H-1152 four-fold [31]. We therefore mutated Ala2016 in LRRK2 to a Thr residue and found that this did not inhibit LRRK2[ G2019S] basal activity, but increased the $\mathrm{IC}_{50}$ of inhibition by $\mathrm{H}-1152 \sim 30$-fold (Fig 5D). We also observed that the LRRK2[ A2016T] mutant was 13 and 12-fold more resistant to inhibition by Y-27632 and sunitinib, respectively (Fig 5D). In the LRRK2[A2016T] mutant the T2016 side chain would clash with these atoms of H-1152, probably forcing it to bind in a rotated and less favourable orientation in the ATP-site. This is the likely explanation of its reduced activity against the mutant compared to the wild-type LRRK2. We also mutated Ala2016 to other residues but found that these mutations markedly inhibited intrinsic LRRK2 activity (data not shown).

Evaluation of ROCK and LRRK2 inhibitors in cells. We next investigated the effect that GSK429286A, GSK269962A, sunitinib, H-1152 and Y-27632 had on phosphorylation of MYPT and ERM proteins in HEK 293 cells. We observed significant basal phosphorylation of MYPT at Thr850 and ERM proteins at site(s) equivalent to Thr567 on ezrin (Fig 6A). In order to activate ROCK we induced stable expression of a constitutively activated G14V-Rho mutant which increased phosphorylation of both MYPT and ezrin $~ 3$-fold (Fig 6A). Treatment of cells with $10 \mu \mathrm{M}$ GSK429286A or GSK269962A, but not sunitinib ablated phosphorylation of MYPT at Thr850 to a similar extent as H-1152 and Y-27632, consistent with ROCK mediating this phosphorylation. However, in the same extracts neither GSK429286A, GSK269962A, sunitinib, H-1152 nor Y-27632 inhibited ERM protein phosphorylation either in the presence or absence of G14V-Rho (Fig 6A). We also found that adding both GSK429286A and sunitinib together to inhibit both LRRK2 and ROCK did not affect basal ERM phosphorylation (Fig 6B).

Immunoprecipitation and assay of endogenous LRRK2. In order to measure protein kinase activity of endogenous LRRK2 (which to our knowledge has previously not been achieved), we generated numerous LRRK2 antibodies and evaluated their ability to immunoprecipitate and immunoblot recombinant full length Flag-LRRK2. This revealed that the antibody raised against a fragment of LRRK2 encompassing amino acids 100-500 immunoprecipitated and immunoblotted Flag-LRRK2 with similar efficiency as anti-Flag antibody (Fig 7A). Moreover, overexpressed FLAG-LRRK2 was immunoprecipitated with the LRRK2 (residues100-500) antibody and possessed similar activity to enzyme immunoprecipitated with Flag-antibody, indicating that the LRRK2 (residues100-500) antibody is not inhibiting LRRK2 protein kinase activity (Fig 7A). We next attempted to immunoprecipitate endogenous LRRK2 from extracts derived from a panel of cell lines using the LRRK2 (residues100-500) antibody. This revealed that Swiss3T3 fibroblasts and RAW macrophages express detectable levels of endogenous LRRK2 protein (Fig 7B). The LRRK2 immunoprecipitates derived from Swiss-3T3 cells were subjected to electrophoresis on a polyacrylamide gel. Staining with Colloidal blue revealed a protein migrating with a molecular weight of $\sim 280 \mathrm{kDa}$ which was confirmed to comprise LRRK2 by mass spectroscopy analysis (Fig 7C). We next subjected LRRK2 and control immunoprecipitates derived from Swiss 3T3 and RAW cells to protein kinase assays employing Nictide as a substrate. This revealed significant protein kinase activity with LRRK2 immunoprecipitates, but not with the control immunoprecipitate (Fig 7D). Moreover, the protein kinase activity detected in the LRRK2 immunoprecipitate was suppressed by H-1152 and sunitinib but not by the selective GSK429286A ROCK inhibitor (Fig 7E). This emphasizes that these compounds are also capable of suppressing the activity of full length LRRK2 containing leucine rich repeats. 
shRNA mediated knock-down of endogenous LRRK2. To further establish that we were indeed observing endogenous LRRK2, we generated RAW macrophage cell lines that were stably infected with lentiviral vectors expressing scrambled shRNA as well as two distinct shRNAs targetting LRRK2. This revealed that both shRNA vectors markedly reduced expression of LRRK2 observed in total cell extracts (Fig 7F) as well as immunoprecipitates (Fig 7G) compared to non-infected cells or cells infected with a scrambled shRNA. We also observed a concomitant reduction in LRRK2 activity associated with the immunoprecipitates derived from cell lines expressing LRRK2 targetting shRNA (Fig 7G). 


\section{Discussion}

Our data reveal that LRRK2 tolerates a wider range of amino acids in its substrates compared to some other protein kinases that have strong requirements for specific amino acids within the substrates that they phosphorylate (Fig 1 and 2). Significant substrate specificity preferences are -5(Trp, Arg), -2(Phe, Tyr, His), -1(Tyr, Arg, Trp), +2 (Arg) and +3 (Arg) positions. Importantly our data suggest that LRRK2 has a strong preference for phosphorylating Thr, as mutation of the phosphorylated Thr residue in Nictide to Ser, abolished phosphorylation of the peptide by LRRK2 (Fig 3). Positional scanning peptide library analysis also suggested that LRRK2 poorly tolerated acidic Glu or Asp residues at all positions surrounding the phosphorylation site (Fig 2A). LRRKtide only possesses a single acidic residue (-4Asp), and the only mutation we tested that improved LRRK2 phosphorylation, was mutation of this residue to Ala (Fig 1C). It is possible that this knowledge of substrate specificity of LRRK2 may aid in the identification of LRRK2 substrates and/or potential phosphorylation sites within identified substrates. This analysis has also enabled us to generate the Nictide peptide, a much improved substrate compared to LRRKtide peptide that is widely deployed to assay LRRK2. A key advantage of Nictide is that it can be used at much lower concentrations in kinases assays. We have been able to assay the activity of endogenous LRRK2 using Nictide, with virtually no background activity observed in the control immunoprecipitate (Fig 7E). To our knowledge this is the first time that activity of endogenous LRRK2 has been assessed. When trying to assay activity of endogenous LRRK2 employing LRRKtide at concentrations of $300 \mu \mathrm{M}\left(\mathrm{K}_{\mathrm{m}}\right.$ value), we observed significant background activity in the pre-immune immunoprecipitation (ND data not shown). Assessment of activity of endogenous LRRK2 is important, as it paves the way to study LRRK2 activity in cells/tissues derived from PD patients. It will enable evaluation of whether LRRK2 protein kinase activity is controlled by extracellular agonists and may also help in the screening for inhibitors for LRRK2. We also observed that fusing Nictide to GST, yielded a highly expressed protein in E.coli ( $4 \mathrm{mg} /$ litre) which was efficiently phosphorylated by LRRK2 in vitro at a greater initial rate than GST-ezrin (residues 505-586) or GSTLRRKtide (Fig 2C). GST-Nictide would serve as a good positive control when evaluating efficiency of phosphorylation of LRRK2 substrates that are identified in future studies.

Our analysis reveals that the substrate specificity of LRRK2 is quite distinct from ROCK2. LRRK2 does not phosphorylate MYPT and ROCK2 poorly phosphorylates ezrin. Moreover, mutations in LRRKtide affected phosphorylation by LRRK2 and ROCK2 in different ways. For example, mutation of the +1 position of the LRRKtide peptide from a Leu to Ala abolished ROCK phosphorylation, without affecting LRRK2 phosphorylation. Many LRRKtide mutations enhanced phosphorylation by ROCK but inhibited phosphorylation by LRRK2. Consistent with ROCK2 phosphorylating ezrin poorly in vitro, we also found that in vivo various ROCK inhibitors failed to inhibit ERM phosphorylation under conditions which they suppressed MYPT phosphorylation. This is consistent with other studies where the Y27632 ROCK inhibitor was found not to suppress ERM phosphorylation [32, 33]. Taken together this data casts doubt on earlier suggestions that ERM proteins are physiologically phosphorylated by ROCK isoforms.

The finding that the H-1152, Y-27632 and sunitinib failed to suppress ERM phosphorylation indicates that either LRRK2 does not phosphorylate ERM in 293 cells or that LRRK2 is not the sole kinase that phosphorylates ERM proteins. As we were unable to detect significant levels of endogenous LRRK2 in 293 cells (Fig 7), we overexpressed LRRK2 and LRRK2[ G2019S] in 293 cells, but this also failed to induce phosphorylation of ERM proteins (JN data not shown). Taken together, this suggests that although ERM proteins are efficiently phosphorylated by LRRK2 in vitro, there is no strong evidence that ERM proteins comprise physiological substrates for LRRK2. Recent studies in Drosophila [34, 35] and primary mouse lymphocytes [36] have suggested that the SLK/LOK STE20 protein kinase might be a key player in controlling ERM phosphorylation. Consistent with this, ERM phosphorylation is reduced but not abolished in lymphocytes derived from SLK/LOK knockout mice [36]. It was shown in 1994 that SLK has an optimal motif of R-R/K$\mathrm{F}-\mathrm{G}-\mathrm{S} / \mathrm{T}-\mathrm{L}-\mathrm{R}-\mathrm{R}-\mathrm{F} / \mathrm{I}$ [37], resembling the ERM site $\mathrm{D}-\mathrm{K}-\mathrm{Y}-\mathrm{K}-\mathrm{T}-\mathrm{L}-\mathrm{R}-\mathrm{Q}-\mathrm{I}$ and is also remarkably similar to the optimal substrate specificity of LRRK2, 
$\mathrm{W}-\mathrm{R}-\mathrm{F}-\mathrm{Y}-\mathrm{T}-\mathrm{L}-\mathrm{R}-\mathrm{R}-\mathrm{A}$. It would also be interesting to test whether residual ERM phosphorylation observed in the SLK/LOK knockout cells was further reduced by treatment with sunitinib and Y-27632 LRRK2 inhibitors. Another recent study has indicated that another STE20 family kinase termed Mst4 kinase can phosphorylate ezrin in polarised epithelial cells in a pathway controlled by the LKB1 tumour suppressor [38]. MST4 was present in our kinase-profiling panel and was not inhibited by H-1152, Y-27632 or sunitinib (Table 1).

The finding that widely utilised ROCK inhibitor Y-27632 (used in $>1400$ papers) as well as H-1152 and hydroxyfasudil inhibit recombinant as well as endogenous LRRK2 with similar potency to that which they target ROCK2 was unexpected, as LRRK2 and ROCK2 are not closely related kinases. LRRK2 lies within the tyrosine-like kinases of the human kinome whilst ROCK2 belongs to the distinct AGC branch [39]. It is therefore possible that some of the physiological effects observed with these ROCK inhibitors could result from inhibition of LRRK2 rather than ROCK isoforms. Identification of GSK429286A is significant, in that it is not only more specific than Y-27632 as assessed on our kinase specificity panel, but also does not significantly inhibit LRRK2 even at doses as high as $30 \mu \mathrm{M}$ (500-fold higher than $\mathrm{IC}_{50}$ of inhibition of ROCK2). GSK429286A will be a useful reagent to use in addition to Y27632 to assess cellular roles of ROCK isoforms. The finding that the LRRK2[ G2019S] mutant was 2 to 4 -fold more sensitive H-1152, Y-27632 and sunitinib than the wild type LRRK2, also indicates that it may be possible to develop compounds that have greater potency towards the Parkinson's disease mutant. It has also been reported that the LRRK2[G2019S] and LRRK2[I202T] mutants that possess elevated activity were also moderately more sensitive to a panel of non-selective kinase inhibitors [40]. If compounds that specifically inhibited Parkinson's disease mutant forms of LRRK2 could be elaborated, they might have lower side effects and not suppress the normal functions of wild type LRRK2. In drug discovery screens being undertaken to identify LRRK2 inhibitors, it could be beneficial to screen compounds against both mutant and wild type forms of LRRK2.

Molecular modelling of the kinase domain of LRRK2 and comparing it with the structures of other kinases revealed a model of how LRRK2 might interact with $\mathrm{H}-1152$. Several residues in the active site of ROCK that are key for binding to H-1152 are also conserved in LRRK2. These include Ala2016, the equivalent of Ala215 in ROCK2 that plays an important role in mediating binding to the inhibitor [30]. Mutation of Ala2016 in LRRK2 to a Thr residue, equivalent to Thr182 in PKA that is weakly inhibited by H-1152, did not affect the basal LRRK2 activity, but markedly suppressed inhibition of LRRK2 by H-1152 and other ROCK kinase inhibitors. The inhibitor resistant LRRK2[ A2016T] mutant might aid in exploring the physiological roles of LRRK2. The wild type and the LRRK2[ A2016T] mutant could be overexpressed in cells and phosphorylation of any target should be less sensitive to inhibition by H-1152, Y-27632 or sunitinib in the cells overexpressing the drug resistant mutant.

Our findings also provide a pharmacological strategy in which phosphorylation of identified LRRK2 substrates could be validated. We suggest that phosphorylation of a LRRK2 substrate should be suppressed by Y-27632 and H-1152 (dual ROCK and LRRK2 inhibitors) as well as sunitinib (inhibits LRRK2 but not ROCK), but not be affected by GSK429286A (inhibits ROCK but LRRK2). In contrast, ROCK mediated processes should be sensitive to GSK429286A in addition to Y-27632 and H-1152, but should not be inhibited by sunitinib. Consistent with this, sunitinib does not inhibit the phosphorylation of MYPT at Thr850 under conditions where this phosphorylation is inhibited by GSK429286A, Y-27632 and H-1152 (Fig 6).

In conclusion, we have undertaken basic analysis of the LRRK2 substrate specificity and developed improved assays to isolate and assess its activity. This will aid in assessing how LRRK2 is regulated and might also facilitate identification of LRRK2 inhibitors that might have potential for treatment of Parkinson's disease. We have also developed a strategy making use of Y-276332 or H-1152, sunitinib and GSK429286A to explore the roles of the LRRK2 kinase. Finally, we recommend that effects prescribed to ROCK based mainly on the 
use of Y-276332 and/or H-1152 be re-evaluated with the more selective GSK429286A inhibitor to ensure that these are indeed mediated by ROCK and not LRRK2.

\section{Acknowledgments}

We thank David Campbell for undertaking mass spectroscopy analysis as well as the Sequencing Service (School of Life Sciences, University of Dundee, Scotland) for DNA sequencing, the Post Genomics and Molecular Interactions Centre for Mass Spectrometry facilities (School of Life Sciences, University of Dundee, Scotland) and the protein production and antibody purification teams [Division of Signal Transduction Therapy (DSTT), University of Dundee] co-ordinated by Hilary McLauchlan and James Hastie for expression and purification of antibodies and Christian Peifer for the inhibitor structures. We would also like to thank Miratul Muqit, Andrew Eatherton, David Livermore, and Dennis Lee for helpful discussions. We thank the Medical Research Council, The Michael J Fox foundation and the pharmaceutical companies supporting the Division of Signal Transduction Therapy Unit (AstraZeneca, Boehringer-Ingelheim, GlaxoSmithKline, Merck \& Co. Inc, Merck KgaA and Pfizer) for financial support. ND is supported by MRCT Industry collaborative award. Lewis Cantley was supported by an NIH grant GM56203. 


\section{References}

1 Zimprich, A., Biskup, S., Leitner, P., Lichtner, P., Farrer, M., Lincoln, S., Kachergus, J., Hulihan, M., Uitti, R. J., Calne, D. B., Stoessl, A. J., Pfeiffer, R. F., Patenge, N., Carbajal, I. C., Vieregge, P., Asmus, F., Muller-Myhsok, B., Dickson, D. W., Meitinger, T., Strom, T. M., Wszolek, Z. K. and Gasser, T. (2004) Mutations in LRRK 2 cause autosomal-dominant parkinsonism with pleomorphic pathology. Neuron 44, 601-607

2 Paisan-Ruiz, C., Jain, S., Evans, E. W., Gilks, W. P., Simon, J., van der Brug, M., Lopez de Munain, A., Aparicio, S., Gil, A. M., Khan, N., Johnson, J., Martinez, J. R., Nicholl, D., Carrera, I. M., Pena, A. S., de Silva, R., Lees, A., Marti-Masso, J. F., Perez-Tur, J., Wood, N. W. and Singleton, A. B. (2004) Cloning of the gene containing mutations that cause PARK8-linked Parkinson's disease. Neuron 44, 595 600

3 Healy, D. G., Falchi, M., O'Sullivan, S. S., Bonifati, V., Durr, A., Bressman, S., Brice, A., Aasly, J., Zabetian, C. P., Goldwurm, S., Ferreira, J. J., Tolosa, E., Kay, D. M., Klein, C., Williams, D. R., Marras, C., Lang, A. E., Wszolek, Z. K., Berciano, J., Schapira, A. H., Lynch, T., Bhatia, K. P., Gasser, T., Lees, A. J. and Wood, N. W. (2008) Phenotype, genotype, and worldwide genetic penetrance of LRRK2-associated Parkinson's disease: a case-control study. Lancet Neurol 7, 583-590

Mata, I. F., Wedemeyer, W. J., Farrer, M. J., Taylor, J. P. and Gallo, K. A. (2006)

LRRK2 in Parkinson's disease: protein domains and functional insights. Trends

Neurosci 29, 286-293

5 Biskup, S. and West, A. B. (2008) Zeroing in on LRRK2-linked pathogenic mechanisms in Parkinson's disease. Biochim Biophys Acta

Greggio, E. and Cookson, M. R. (2009) Leucine Rich Repeat Kinase 2 mutations and Parkinson's disease: Three Questions. ASN Neuro In Press

Covy, J. P. and Giasson, B. I. (2009) Identification of compounds that inhibit the kinase activity of leucine-rich repeat kinase 2. Biochem Biophys Res Commun 378, 473-477

Anand, V. S., Reichling, L. J., Lipinski, K., Stochaj, W., Duan, W., Kelleher, K., Pungaliya, P., Brown, E. L., Reinhart, P. H., Somberg, R., Hirst, W. D., Riddle, S. M. and Steven, P. B. (2009) Investigation of leucine-rich repeat kinase 2: enzymological properties and novel assays. Febs $\mathbf{J} 276,466-478$

9 Jaleel, M., Nichols, R. J., Deak, M., Campbell, D. G., Gillardon, F., Knebel, A. and Alessi, D. R. (2007) LRRK2 phosphorylates moesin at threonine-558:

characterization of how Parkinson's disease mutants affect kinase activity. Biochem $\mathrm{J}$ 405, 307-317

10 Matsui, T., Maeda, M., Doi, Y., Yonemura, S., Amano, M., Kaibuchi, K., Tsukita, S. and Tsukita, S. (1998) Rho-kinase phosphorylates COOH-terminal threonines of ezrin/radixin/moesin (ERM) proteins and regulates their head-to-tail association. J Cell Biol 140, 647-657

11 Oshiro, N., Fukata, Y. and Kaibuchi, K. (1998) Phosphorylation of moesin by rhoassociated kinase (Rho-kinase) plays a crucial role in the formation of microvilli-like structures. J Biol Chem 273, 34663-34666

12 Tran Quang, C., Gautreau, A., Arpin, M. and Treisman, R. (2000) Ezrin function is required for ROCK-mediated fibroblast transformation by the Net and Dbl oncogenes. Embo J 19, 4565-4576

13 Goodman, K. B., Cui, H., Dowdell, S. E., Gaitanopoulos, D. E., Ivy, R. L., Sehon, C. A., Stavenger, R. A., Wang, G. Z., Viet, A. Q., Xu, W., Ye, G., Semus, S. F., Evans, C., Fries, H. E., Jolivette, L. J., Kirkpatrick, R. B., Dul, E., Khandekar, S. S., Yi, T., Jung, D. K., Wright, L. L., Smith, G. K., Behm, D. J., Bentley, R., Doe, C. P., Hu, E. and Lee, D. (2007) Development of dihydropyridone indazole amides as selective Rho-kinase inhibitors. J Med Chem 50, 6-9

14 Biskup, S., Moore, D. J., Rea, A., Lorenz-Deperieux, B., Coombes, C. E., Dawson, V. L., Dawson, T. M. and West, A. B. (2007) Dynamic and redundant regulation of LRRK2 and LRRK1 expression. BMC Neurosci 8, 102 
15 Sward, K., Dreja, K., Susnjar, M., Hellstrand, P., Hartshorne, D. J. and Walsh, M. P. (2000) Inhibition of Rho-associated kinase blocks agonist-induced Ca2+ sensitization of myosin phosphorylation and force in guinea-pig ileum. J Physiol 522 Pt 1, 33-49

16 Feng, J., Ito, M., Ichikawa, K., Isaka, N., Nishikawa, M., Hartshorne, D. J. and Nakano, T. (1999) Inhibitory phosphorylation site for Rho-associated kinase on smooth muscle myosin phosphatase. J Biol Chem 274, 37385-37390

17 Hutti, J. E., Jarrell, E. T., Chang, J. D., Abbott, D. W., Storz, P., Toker, A., Cantley, L. C. and Turk, B. E. (2004) A rapid method for determining protein kinase phosphorylation specificity. Nat Methods 1, 27-29

18 Turk, B. E., Hutti, J. E. and Cantley, L. C. (2006) Determining protein kinase substrate specificity by parallel solution-phase assay of large numbers of peptide substrates. Nat Protoc 1, 375-379

19 Workman, C. T., Yin, Y., Corcoran, D. L., Ideker, T., Stormo, G. D. and Benos, P. V. (2005) enoLOGOS: a versatile web tool for energy normalized sequence logos. Nucleic Acids Res 33, W389-392

20 Hutti, J. E., Turk, B. E., Asara, J. M., Ma, A., Cantley, L. C. and Abbott, D. W. (2007) IkappaB kinase beta phosphorylates the K63 deubiquitinase A20 to cause feedback inhibition of the NF-kappaB pathway. Mol Cell Biol 27, 7451-7461

21 Miller, M. L., Jensen, L. J., Diella, F., Jorgensen, C., Tinti, M., Li, L., Hsiung, M., Parker, S. A., Bordeaux, J., Sicheritz-Ponten, T., Olhovsky, M., Pasculescu, A., Alexander, J., Knapp, S., Blom, N., Bork, P., Li, S., Cesareni, G., Pawson, T., Turk, B. E., Yaffe, M. B., Brunak, S. and Linding, R. (2008) Linear motif atlas for phosphorylation-dependent signaling. Sci Signal 1, ra2

22 Ishizaki, T., Uehata, M., Tamechika, I., Keel, J., Nonomura, K., Maekawa, M. and Narumiya, S. (2000) Pharmacological properties of Y-27632, a specific inhibitor of rho-associated kinases. Mol Pharmacol 57, 976-983

23 Shimokawa, H., Seto, M., Katsumata, N., Amano, M., Kozai, T., Yamawaki, T., Kuwata, K., Kandabashi, T., Egashira, K., Ikegaki, I., Asano, T., Kaibuchi, K. and Takeshita, A. (1999) Rho-kinase-mediated pathway induces enhanced myosin light chain phosphorylations in a swine model of coronary artery spasm. Cardiovasc Res 43, 1029-1039

24 Sasaki, Y., Suzuki, M. and Hidaka, H. (2002) The novel and specific Rho-kinase inhibitor (S)-(+)-2-methyl-1-[(4-methyl-5-isoquinoline)sulfonyl]-homopiperazine as a probing molecule for Rho-kinase-involved pathway. Pharmacol Ther 93, 225-232

25 Tamura, M., Nakao, H., Yoshizaki, H., Shiratsuchi, M., Shigyo, H., Yamada, H., Ozawa, T., Totsuka, J. and Hidaka, H. (2005) Development of specific Rho-kinase inhibitors and their clinical application. Biochim Biophys Acta 1754, 245-252

26 Chijiwa, T., Mishima, A., Hagiwara, M., Sano, M., Hayashi, K., Inoue, T., Naito, K., Toshioka, T. and Hidaka, H. (1990) Inhibition of forskolin-induced neurite outgrowth and protein phosphorylation by a newly synthesized selective inhibitor of cyclic AMP-dependent protein kinase, N-[2-(p-bromocinnamylamino)ethyl]-5isoquinolinesulfonamide (H-89), of PC12D pheochromocytoma cells. J Biol Chem 265, 5267-5272

27 Shibuya, M., Suzuki, Y., Sugita, K., Saito, I., Sasaki, T., Takakura, K., Nagata, I., Kikuchi, H., Takemae, T., Hidaka, H. and et al. (1992) Effect of AT877 on cerebral vasospasm after aneurysmal subarachnoid hemorrhage. Results of a prospective placebo-controlled double-blind trial. J Neurosurg 76, 571-577

28 Doe, C., Bentley, R., Behm, D. J., Lafferty, R., Stavenger, R., Jung, D., Bamford, M., Panchal, T., Grygielko, E., Wright, L. L., Smith, G. K., Chen, Z., Webb, C., Khandekar, S., Yi, T., Kirkpatrick, R., Dul, E., Jolivette, L., Marino, J. P., Jr., Willette, R., Lee, D. and Hu, E. (2007) Novel Rho kinase inhibitors with antiinflammatory and vasodilatory activities. J Pharmacol Exp Ther 320, 89-98

29 Yamaguchi, H., Kasa, M., Amano, M., Kaibuchi, K. and Hakoshima, T. (2006) Molecular mechanism for the regulation of rho-kinase by dimerization and its inhibition by fasudil. Structure 14, 589-600

30 Jacobs, M., Hayakawa, K., Swenson, L., Bellon, S., Fleming, M., Taslimi, P. and Doran, J. (2006) The structure of dimeric ROCK I reveals the mechanism for ligand selectivity. J Biol Chem 281, 260-268 
31 Bonn, S., Herrero, S., Breitenlechner, C. B., Erlbruch, A., Lehmann, W., Engh, R. A., Gassel, M. and Bossemeyer, D. (2006) Structural analysis of protein kinase A mutants with Rho-kinase inhibitor specificity. J Biol Chem 281, 24818-24830

32 Matsui, T., Yonemura, S., Tsukita, S. and Tsukita, S. (1999) Activation of ERM proteins in vivo by Rho involves phosphatidyl-inositol 4-phosphate 5-kinase and not ROCK kinases. Curr Biol 9, 1259-1262

33 Lee, J. H., Katakai, T., Hara, T., Gonda, H., Sugai, M. and Shimizu, A. (2004) Roles of p-ERM and Rho-ROCK signaling in lymphocyte polarity and uropod formation. J Cell Biol 167, 327-337

34 Carreno, S., Kouranti, I., Glusman, E. S., Fuller, M. T., Echard, A. and Payre, F. (2008) Moesin and its activating kinase Slik are required for cortical stability and microtubule organization in mitotic cells. J Cell Biol 180, 739-746

35 Kunda, P., Pelling, A. E., Liu, T. and Baum, B. (2008) Moesin controls cortical rigidity, cell rounding, and spindle morphogenesis during mitosis. Curr Biol 18, 91101

36 Belkina, N. V., Liu, Y., Hao, J. J., Karasuyama, H. and Shaw, S. (2009) LOK is a major ERM kinase in resting lymphocytes and regulates cytoskeletal rearrangement through ERM phosphorylation. Proc Natl Acad Sci U S A 106, 4707-4712

37 Songyang, Z., Blechner, S., Hoagland, N., Hoekstra, M. F., Piwnica-Worms, H. and Cantley, L. C. (1994) Use of an oriented peptide library to determine the optimal substrates of protein kinases. Curr Biol 4, 973-982

38 ten Klooster, J. P., Jansen, M., Yuan, J., Oorschot, V., Begthel, H., Di Giacomo, V., Colland, F., de Koning, J., Maurice, M. M., Hornbeck, P. and Clevers, H. (2009) Mst4 and Ezrin induce brush borders downstream of the Lkb1/Strad/Mo25 polarization complex. Dev Cell 16, 551-562

39 Manning, G., Whyte, D. B., Martinez, R., Hunter, T. and Sudarsanam, S. (2002) The protein kinase complement of the human genome. Science 298, 1912-1934

40 Reichling, L. J. and Riddle, S. M. (2009) Leucine-rich repeat kinase 2 mutants I2020T and G2019S exhibit altered kinase inhibitor sensitivity. Biochem Biophys Res Commun 384, 255-258 


\section{Figure Legends}

Figure 1 Comparison of ROCK substrates as substrates for LRRK2 (A-upper panel) HisROCK2 (residues 2-543) and GST-LRRK2[G2019S] (residues 1326-2527) were diluted to a concentration where they possessed identical activity towards LRRKtide and then incubated with $2 \mu \mathrm{M}$ MBP-MYPT (residues 714-1004) or heat treated GST-Ezrin (full-length) [9] in the presence of $\mathrm{Mg}\left[\gamma_{-}{ }^{32} \mathrm{P}\right]$-ATP. Enzyme was decreased successively two fold, represented by decreasing slope in triangle. Reactions were terminated after $15 \mathrm{~min}$ by addition of sample buffer and products were subjected to SDS-PAGE. Gels were analysed by staining with colloidal blue (CB) and phosphorylation was monitored by autoradiography. Immunoblotting analysis was also undertaken with the indicated antibodies. (A-lower panel) Comparison of the amino acid sequences surrounding Thr850-MYPT and Thr567-ezrin showing little similarity between peptides. (B) Sequence alignments of the indicated species of ERM proteins surrounding the ROCK/LRRK2 phosphorylation site. Identical residues are shaded. The accession sequences used were: human moesin [accession no. NP_002435] human ezrin [accession no. NP 03370] human radixin [accession no. NP 002897]. Mus musculus [accession no. NP ${ }^{-}$034963], Danio rerio [accession no. NP 001004296], Drosophila melanogaster (Dm) [accession no. NP_727290], Caenorhabditis elegans (Ce) [accession no. NP491550]. (C) Analysis of substrate recognition determinants in LRRKtide. Residues from -6 to -5 of the LRRKtide substrate (RLGRDKYKTLRQIRQ) were mutated to the residue indicated in bold lettering. These peptides were analyzed for their ability to be phosphorylated by GST-LRRK2 [ G2019S] (residues 1326-2527) purified from HEK293 cells or ROCK2 (residues 2-543) purified from baculovirus. NP denotes that the peptide was phosphorylated poorly and that kinetic analysis was not feasible. For $\mathrm{K}_{\mathrm{m}}$ values above 500 $\mu \mathrm{M}$ an " $\sim$ " sign is added to stress that these values were inferred from kinetic analysis undertaken at peptide concentrations of lower than $1 \mathrm{mM}$. Similar results were obtained in at least two experiments.

\section{Figure 2. Determination of the preferred substrate phosphorylation sequence for}

\section{LRRK2. (A) Recombinant HEK293 purified GST-LRRK2[G2019S] (residues 1326-2527)} and catalytically inactive GST-LRRK2[D2017A] (residues 1326-2527) was used to screen a positional scanning peptide library consisting of 189 biotinylated-peptide libraries in individual kinase assays. Reaction products were bound to streptavidin coated membrane and after washing, phosphorylation was visualised by phosphorimaging. (B) Logo of LRRK2 phosphorylation site was derived from empirical data from (A) input into enologos ${ }^{\circledR}$. The height of the stack of single amino acid letters indicates the entropy of the site and the size of each letter indicates its preference at the position relative to the phosphorylation site between -5 and +4 . The largest letters at each position in the logo were chosen to substitute for residues in a longer version of the LRRKtide substrate peptide to derive Nictide, shown below the logo. (C) GST fusion proteins with the indicated peptide sequences of LRRKtide, the longer LRRKtide, the carboxy terminus of moesin (residues 500-577) and the Nictide substrates were subjected to phosphorylation by HEK293 purified LRRK2[G2019S] (residues 1326-2527). Reactions were stopped by the addition of sample buffer and products were subjected to SDS-PAGE. Gels were analysed by staining with colloidal blue (CB) and phosphorylation was monitored by autoradiography $\left({ }^{32} \mathrm{P}\right)$. Similar results were obtained in replicate experiments.

\section{Figure 3. Kinetic analysis of the Nictide Substrate.}

(A). Residues from -5 to +5 of the Nictide substrate (RLGWWRFYTLRRARQGNTKQR) were mutated to the residue indicated in bold lettering. These peptides were analyzed for their ability to be phosphorylated by GST-LRRK2[G2019S] (residues 1326-2527) or GSTLRRK2[Wild type] (residues 1326-2527) purified from HEK293 cells and $K_{\mathrm{m}}$ and $V_{\max }$ values were derived by nonlinear regression analysis as described in Materials and Methods. Similar results were obtained in at least three experiments. (B). Average values from a representative experiment from which data in A were derived for GST-LRRK2[G2019S] (residues1326-2527). (C). As in B. except with or GST-LRRK2[wild type] (residues13262527). 
Figure 4. Characterization of ROCK inhibitors as LRRK2 inhibitors. (A). The chemical structures of the inhibitors utilized in this study. (B) GST-LRRK2[wild type] (residues 13262527) or GST-[G2019S] (residues 1326-2527) or His-ROCK2 (residues 2-543) were assayed in the presence or absence of the indicated concentration of the indicated inhibitor, in the presence of $100 \mu \mathrm{M}$ ATP. The results are presented as percentage of kinase activity relative to the control measured in the presence of DMSO. Results are the average of at least duplicate reactions where similar results were observed in at least one other experiment. The inhibitors are represented as follows: H89 by black line and circles; fasudil by red line squares; hydroxyfasudil by bright green line and triangles; Y-27632 by blue line and inverted triangles; H-1152 by purple diamonds; GSK429286A by orange circles; GSK269962A by dark green squares; and sunitinib by magenta triangles. (C) The $\mathrm{IC}_{50}$ values derived from the graphs in (B) are shown in $\mu \mathrm{M}$ and displayed in tabular format.

Figure 5. Design of an LRRK2 inhibitor desensitized mutant. (A) Structural model of the LRRK2 kinase domain in complex with $\mathrm{H}-1152$ based on a ROCK crystal structure. The predicted positions of A1950, A2016 and D2017 are indicated. The right panel is rotated slightly. (B) Sequence alignment of the amino acids surrounding the Asp of subdomain VII for LRRK2, Protein Kinase A (PKA) and ROCK2. Identical amino acids are shaded in black and similar amino acids are shaded in grey. Terminal amino acid residues are numbered. (C) GST-LRRK2[G2019S] (residues 1326-2527) and GST-LRRK2[G2019S/A2016T] (residues 1326-2527) were assayed in the presence or absence of the indicated concentration of the inhibitor, in the presence of $100 \mu \mathrm{M}$ ATP and expressed as a percent of control reactions performed in the presence of vehicle alone. GST-LRRK2[G2019S] (residues 1326-2527) reactions are represented by filled circles and GST-LRRK2[G2019S/A2016T] (residues 1326-2527) reactions are represented by open circles. Colour scheme is the same as in Figure $4 \mathrm{~B}$.

\section{Figure 6. Testing the efficacy of LRRK2 and ROCK inhibitors in vivo.}

(A) Flp-in T-REx cells that harbor GFP tagged constitutively active G14V-Rho were either left uninduced or induced by the inclusion of $1 \mu \mathrm{g} / \mathrm{ml}$ doxycycline in the culture medium. At 7 hours post induction, Cells were treated with 10uM GSK429286A, GSK269962A, H-1152 and Y-27632 for $1 \mathrm{~h}$. Cells were lysed in direct SDS-Lysis Buffer and resolved on 4-12\% Novex gels and subjected to immunoblot analysis with the indicated antibodies. (B) As in (A) except that HEK 293 cells were used and GSK429286A was used in conjunction with sunitinib.

Figure 7. Analysis of Endogenous LRRK2 (A) Doxycycline inducible HEK 293 cells overexpressing full length flag-tagged human LRRK2 and LRRK2 containing D2017A and G2019S mutations were generated as described in material and methods. Anti-LRRK2 (residues 100-500) S348C antibody was used to retrieve recombinant protein. Precipitates were assayed for kinase activity using LRRKtide and were immunoblotted with anti-FLAG and S374C anti-LRRK2 (residues 2498-2514) (B) Extracts of the indicated cell lines were screened for the presence of LRRK2 protein following immunoprecipitation with the indicated antibodies and immunoblotting with the LRRK2 (residues 2498-2514) S374C antibody. (C) Anti-LRRK2[ 100-500] and control IgG were used in immunoprecipitations of $60 \mathrm{mg}$ Swiss 3T3 lysate. Specific bands corresponding to the predicted molecular weight of LRRK2 were excised and tryptic peptides were identified by mass spectrometry. (D) Endogenous LRRK2 kinase activity from S348C anti-LRRK2[ 100-500] was measured against the Nictide substrate in Swiss 3T3 and RAW cells following immunoprecipitation. (E) Immunoprecipitates from Swiss-3T3 cell lines were assayed against Nictide substrate in the presence of the indicated concentrations of the indicated inhibitors. Kinase assays of immunecomplexes were carried out in triplicate and are representative of at least two seperate experiments. Errors are s.e.m. (F) Lysates of RAW cells, stably transduced with the indicated shRNA expressing lentivirus, were resolved on $8 \%$ SDS-PAGE gels and immunoblotted with the LRRK2 (residues 2498-2514) S374C antibody. Anti- tubulin was used as a loading control. (G) Endogenous LRRK2 kinase activity against Nictide was assayed as in (E), from RAW cells stably transduced with the indicated shRNA expressing lentivirus. 
Table 1. Kinase profiling of LRRK2 and ROCK inhibitors. Results are presented as percentage of kinase activity compared to control incubations in which inhibitor was omitted. Protein kinases were assayed as described at the material and methods section. The results are an average of a triplicate determination \pm standard deviation. Abbreviations not defined in main text: AMPK, AMP-activated protein kinase BRSK, brain-specific kinase; BTK, Bruton's tyrosine kinase; CaMK1, calmodulin-dependent kinase; CaMKK, CaMK kinase; CDK, cyclin-dependent kinase; CHK, checkpoint kinase; CK1, casein kinase 1; CSK, Cterminal Src kinase; DYRK, dual-specificity tyrosine-phosphorylated and regulated kinase; ERK, mitogen activated protein kinase; EF2K, elongation-factor-2 kinase; EPH, ephrin; FGF-R, fibroblast growth factor receptor; GCK, germinal center kinase; GSK3, glycogen synthase kinase 3; HIPK, homeodomain-interacting protein kinase; HER4, V-erb a erythroblastic leukemia viral oncogene homolog 1; IRAK, Interleukin-1 Receptor-Associated Kinase 4; IGF1R, IGF1 receptor; IKK, inhibitory $\kappa B$ kinase; IR, insulin receptor; IRR, insulin-related receptor; JNK, c-Jun N-terminal kinase; Lck, lymphocyte cell-specific protein tyrosine kinase; MAPKAP-K, MAPK-activated protein kinase; MARK, microtubule-affinityregulating kinase; MELK, maternal embryonic leucine-zipper kinase; MKK1, MAPK kinase1; MLCK, smooth muscle myosin light-chain kinase; MNK, MAPK-integrating protein kinase; MLK, mixed lineage kinase; MINK, Misshapen-like Kinase; MSK, mitogen- and stress-activated protein kinase; MST, mammalian homologue Ste20-like kinase; NEK, NIMA (never in mitosis in Aspergillus nidulans)-related kinase; NUAK1, SNF1 like kinase1; PAK, p21-activated protein kinase; PHK, phosphorylase kinase; PIM, provirus integration site for Moloney murine leukaemia virus; PKA, cAMP-dependent protein kinase; PDK1, 3phosphoinositide-dependent protein kinase-1; PKB, protein kinase $\mathrm{B}$; PKC, protein kinase $\mathrm{C}$ PKD, protein kinase D; PLK, polo-like kinase; PRAK, p38-regulated activated kinase; PRK, protein kinase C-related kinase; RSK, ribosomal S6 kinase; S6K, p70 ribosomal S6 kinase; SGK1, serum and glucocorticoid kinase 1; SRPK, serine-arginine protein kinase; SYK, spleen tyrosine kinase; TBK1, TANK-binding kinase 1; TTK, tau-tubulin kinase; VEGFR, vascular endothelial growth factor receptor; YES1, Yamaguchi sarcoma viral oncogene homologue 1. Bold and underline indicates inhibition of $80 \%$ or more. 
Figure 1

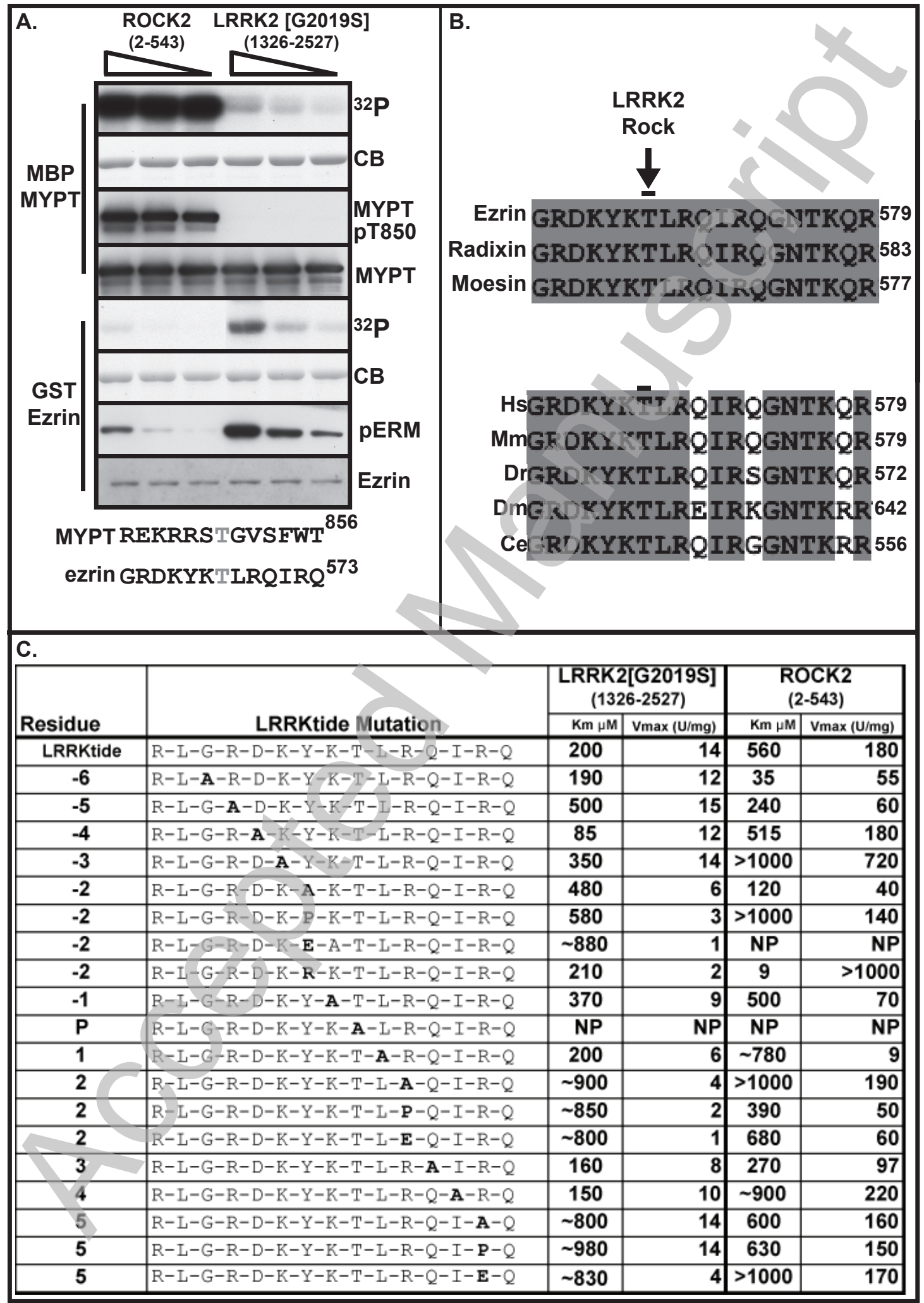

Licenced copy. Copying is not permitted, except with prior permission and as allowed by law.

(C) 2009 The Authors Journal compilation (c) 2009 Portland Press Limited 
FIGURE 2

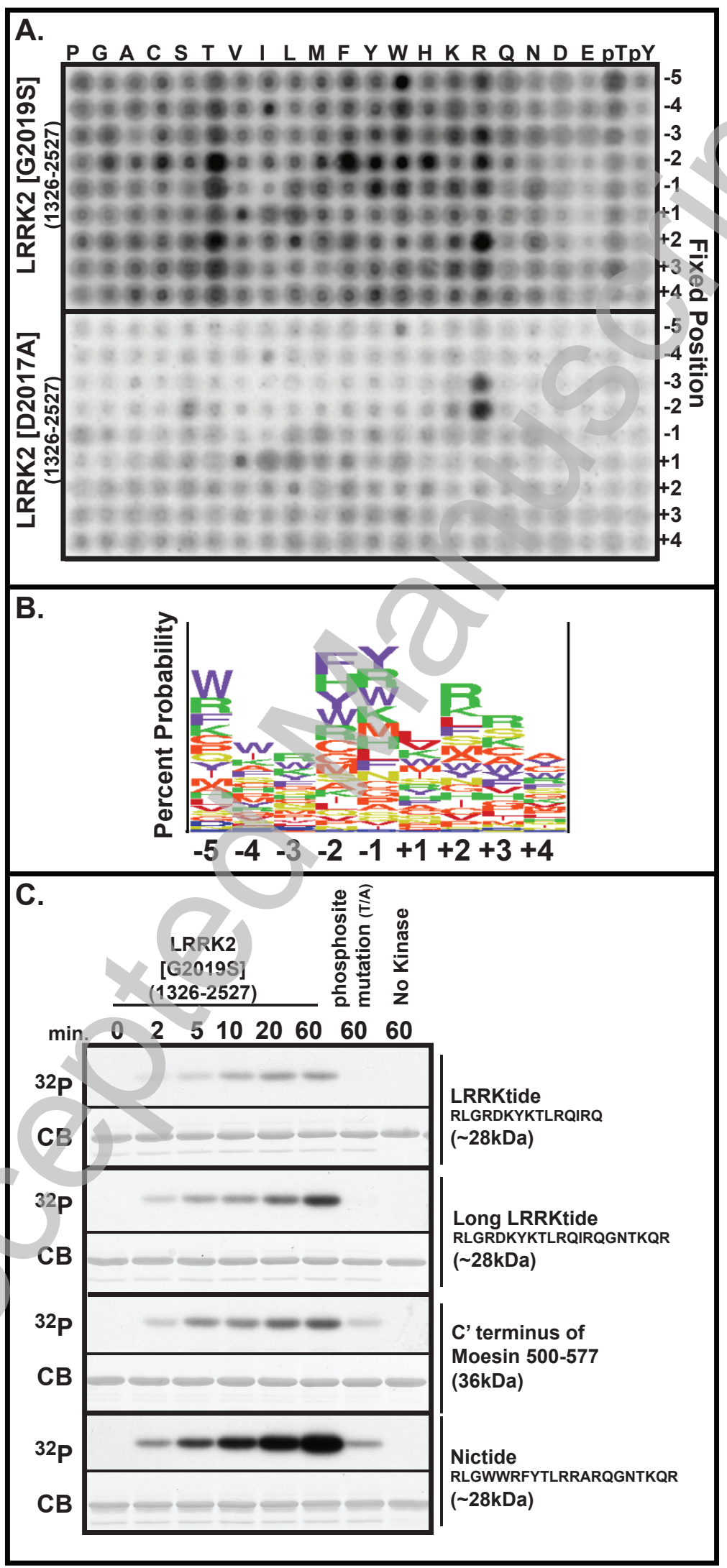

Licenced copy. Copying is not permitted, except with prior permission and as allowed by law. (C) 2009 The Authors Journal compilation (c) 2009 Portland Press Limited 


\section{Figure 3}

\begin{tabular}{|c|c|c|c|c|c|}
\hline \multirow[b]{2}{*}{ Position } & \multirow[b]{2}{*}{ Peptide sequence } & \multicolumn{2}{|c|}{$\begin{array}{c}\text { LRRK2 [G2019S] } \\
(1326-2527)\end{array}$} & \multicolumn{2}{|c|}{\begin{tabular}{|c} 
LRRK2 [Wild type \\
$(1326-2527)$
\end{tabular}} \\
\hline & & $\begin{array}{l}\mathrm{K}_{\mathrm{m}} \\
\mu \mathrm{M}\end{array}$ & $\begin{array}{l}\mathrm{V}_{\max } \\
\mathrm{U} / \mathrm{mg}\end{array}$ & $\begin{array}{l}\mathrm{K}_{\mathrm{m}} \\
\mu \mathrm{M}\end{array}$ & $\begin{array}{l}\mathrm{V}_{\max } \\
\mathrm{U} / \mathrm{mg}\end{array}$ \\
\hline Nictide & RLGWWRFYTLRRARQGNTKQR & 10 & 26 & 9 & 15 \\
\hline-5 & RLGAWRFYTLRRARQGNTKQR & 31 & 56 & 30 & 31 \\
\hline-4 & RLGWARFYTLRRARQGNTKQR & 20 & 40 & 33 & 32 \\
\hline-3 & RLGWWAFYTLRRARQGNTKQR & 10 & 11 & 9 & 5 \\
\hline-2 & RLGWWRAYTLRRARQGNTKQR & 15 & 25 & 19 & 23 \\
\hline-1 & RLGWWRFATLRRARQGNTKQR & 24 & 44 & 17 & 16 \\
\hline $\mathbf{P}$ & RLGWWRFYALRRARQGNTKQR & NP & NP & NP & NP \\
\hline+1 & RLGWWRFYTARRARQGNTKQR & 17 & 23 & 13 & 8 \\
\hline+2 & RLGWWRFYTAARARQGNTKQR & 21 & 12 & 18 & 3 \\
\hline+3 & RLGWWRFYTLAAARQGNTKQR & 18 & 18 & 10 & 5 \\
\hline+5 & RLGWWRFYTLRAAAQGNTKQR & 17 & 22 & 14 & 10 \\
\hline+1 & RLGWWRFYT PRRARQGNTKQR & 8 & 16 & 14 & 8 \\
\hline$-5,-4$ & RLGAARFYTLRRARQGNTKQR & $\sim 100$ & 75 & $\sim 130$ & 70 \\
\hline$-5,-1$ & RLGAWRFATLRRARQGNTKQR & 50 & 60 & $\sim 100$ & 36 \\
\hline$-5,-4,+1$ & RLGAARFYTARRARQGNTKQR & $\sim 230$ & 65 & 18 & 20 \\
\hline-2 & RLGWWRTYTLRRARQGNTKQR & 18 & 18 & 23 & 10 \\
\hline+2 & RLGWWRFYTLTRARQGNTKQR & 25 & 16 & 31 & 9 \\
\hline$-2 \mathrm{~T},+2 \mathrm{~T}$ & RLGWWRTYTLTRARQGNTKQR & $\sim 130$ & 15 & $\sim 130$ & 6 \\
\hline $\mathrm{T} \rightarrow \mathrm{S}$ & RLGWWRFYSLRRARQGNTKQR & NP & NP & NP & NP \\
\hline
\end{tabular}

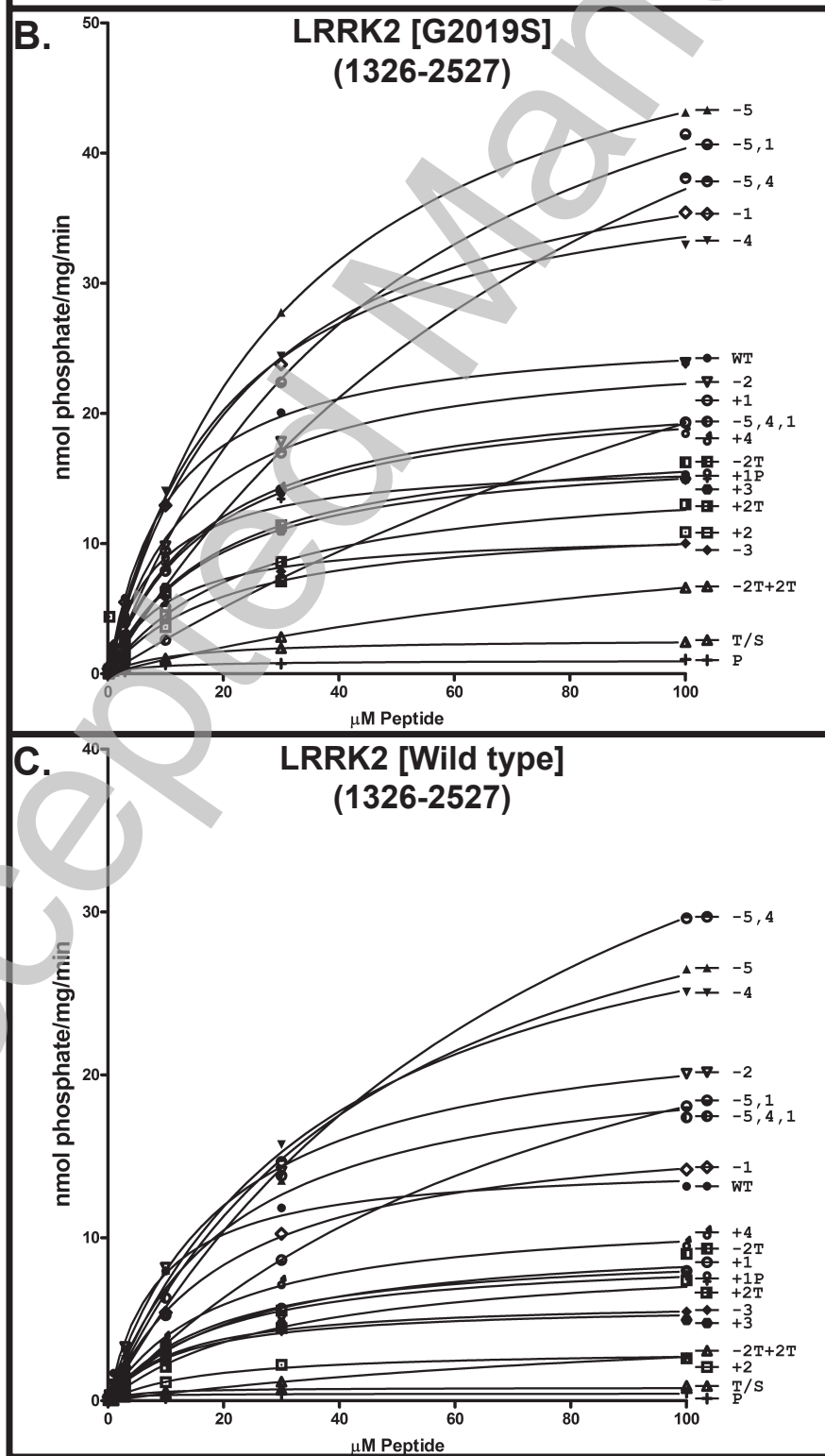

Licenced copy. Copying is not permitted, except with prior permission and as allowed by law. (C) 2009 The Authors Journal compilation (c) 2009 Portland Press Limited 
Biochemical Journal Immediate Publication. Published on 09 Sep 2009 as manuscript BJ20091035

Figure 4

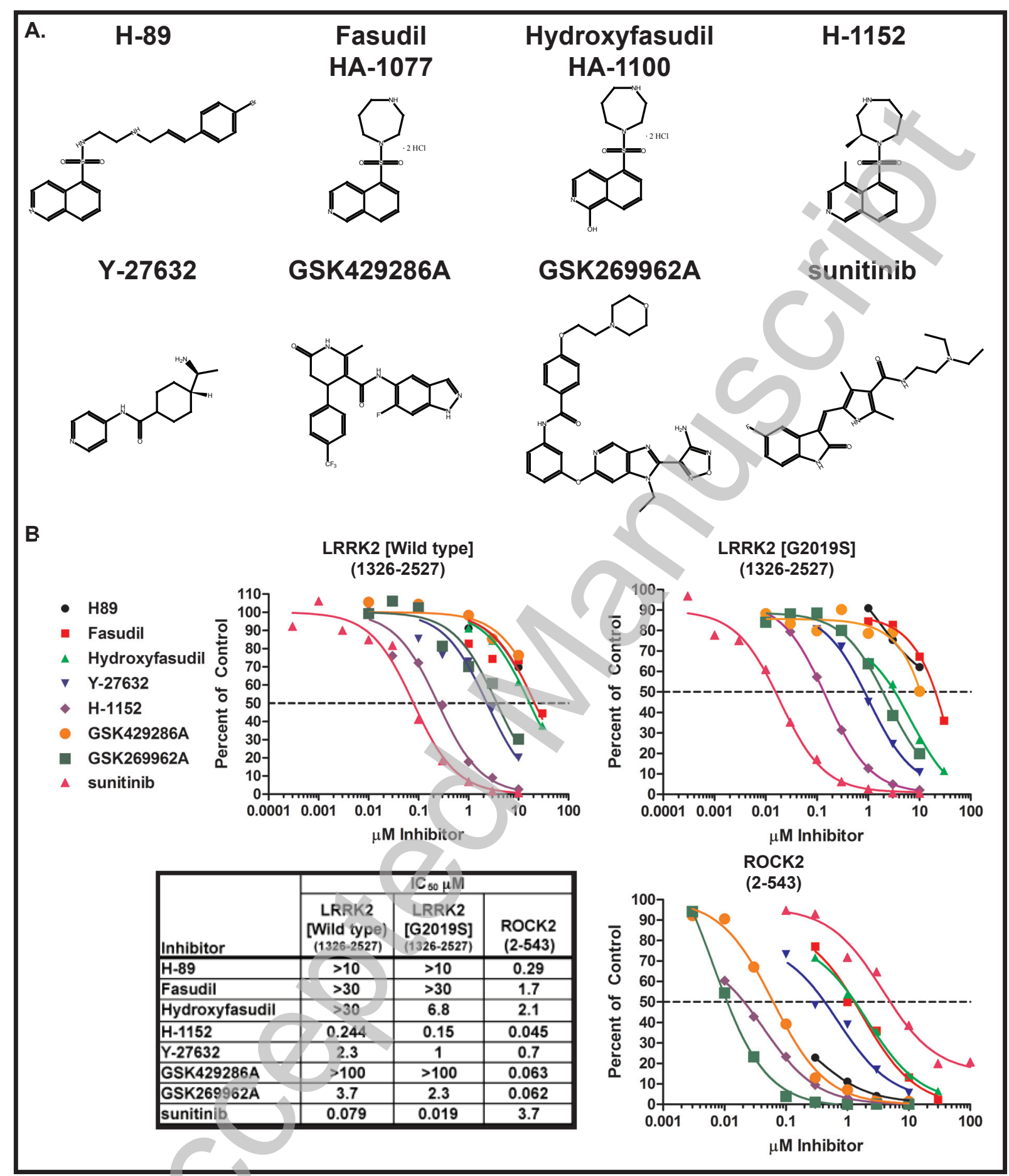

Licenced copy. Copying is not permitted, except with prior permission and as allowed by law.

(C) 2009 The Authors Journal compilation (c) 2009 Portland Press Limited 
Biochemical Journal Immediate Publication. Published on 09 Sep 2009 as manuscript BJ20091035

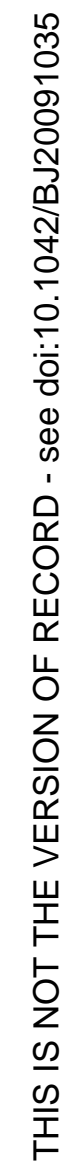

Figure 5

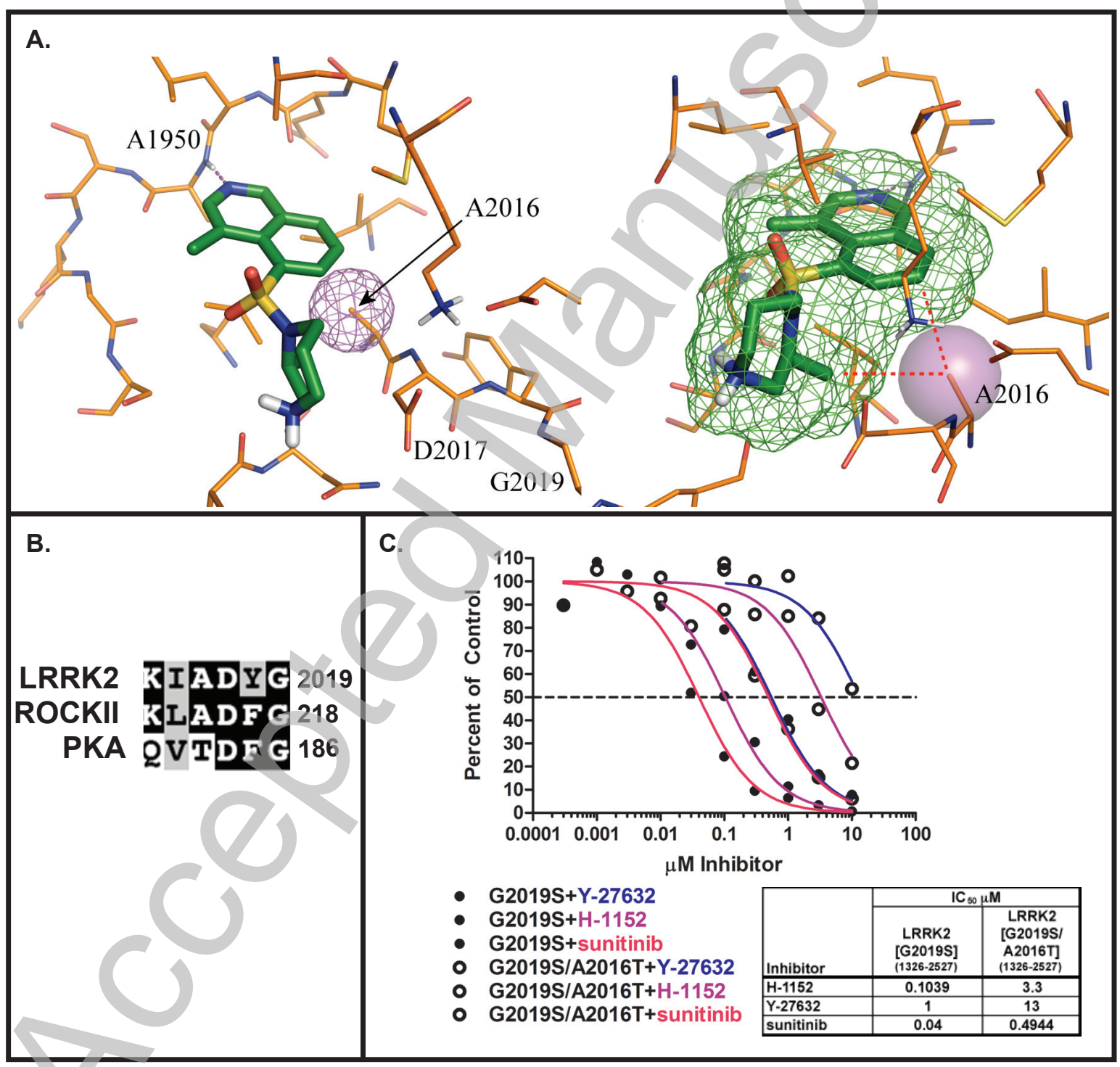

Licenced copy. Copying is not permitted, except with prior permission and as allowed by law. (C) 2009 The Authors Journal compilation (c) 2009 Portland Press Limited 
Biochemical Journal Immediate Publication. Published on 09 Sep 2009 as manuscript BJ20091035

Figure 6

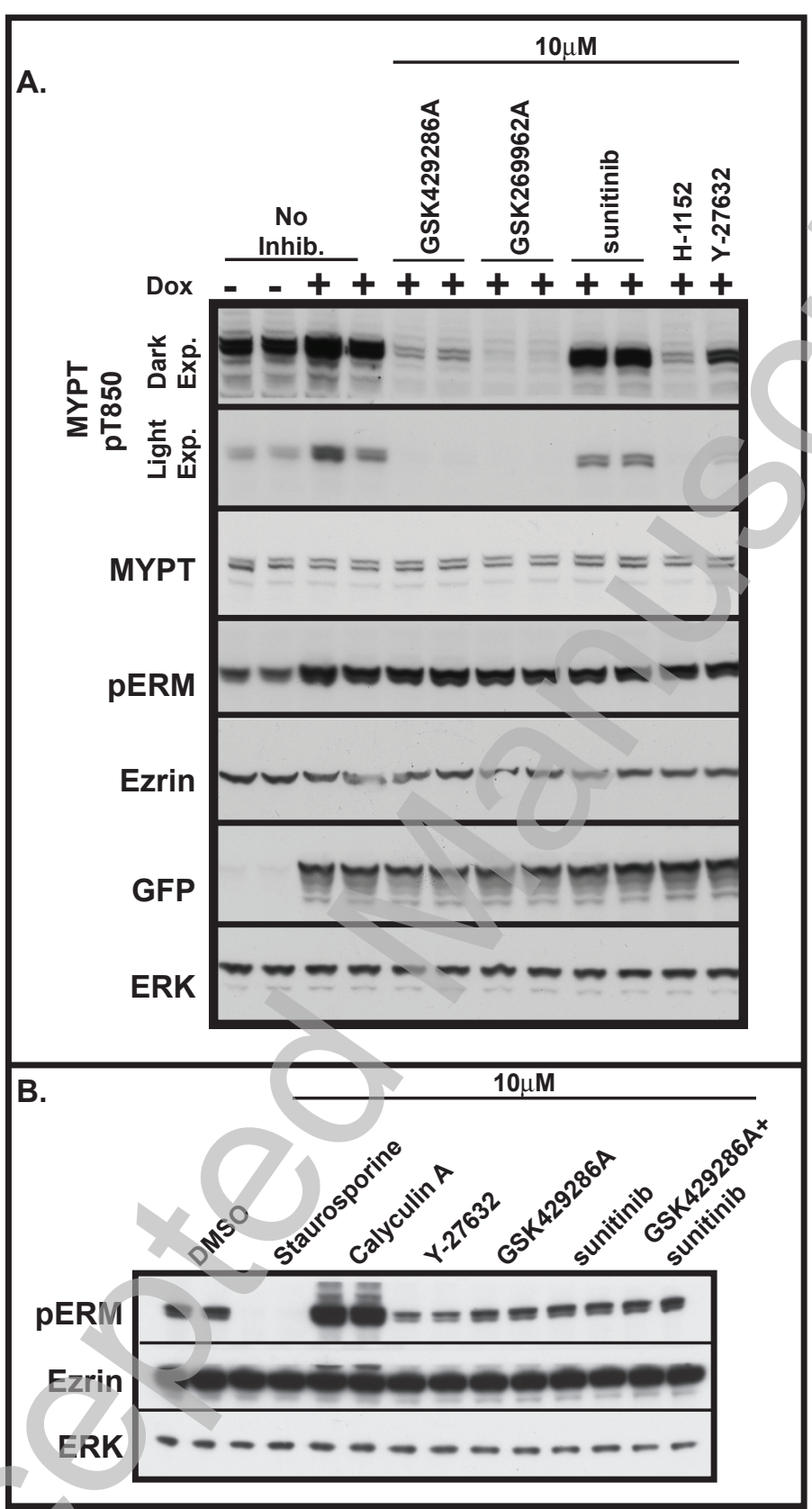

Licenced copy. Copying is not permitted, except with prior permission and as allowed by law. (c) 2009 The Authors Journal compilation (c) 2009 Portland Press Limited 
Figure 7

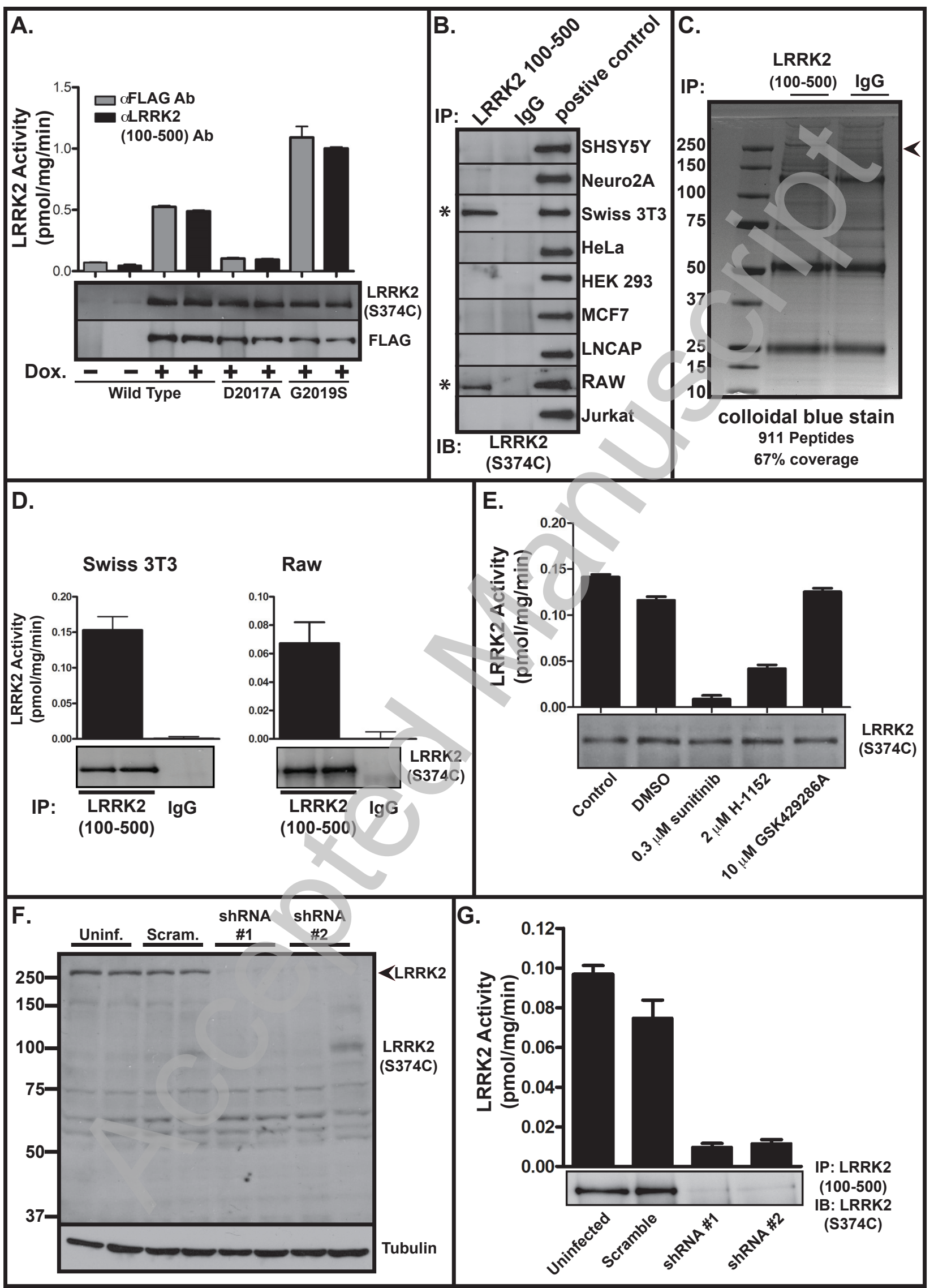

Licenced copy. Copying is not permitted, except with prior permission and as allowed by law. (C) 2009 The Authors Journal compilation (c) 2009 Portland Press Limited 
TABLE 1

\begin{tabular}{|c|c|c|c|c|c|c|c|c|c|}
\hline & & $\begin{array}{c}\text { HA-1100 } \\
10 u M\end{array}$ & $\begin{array}{c}\text { HA-1077 } \\
10 \mathrm{uM}\end{array}$ & $\begin{array}{c}\mathrm{H}-1152 \\
1 \mathrm{uM}\end{array}$ & $\begin{array}{c}\text { Y-27632 } \\
10 \mathrm{uM} \\
\end{array}$ & $\begin{array}{c}\text { GSK429286A } \\
1 \mathrm{uM} \\
\end{array}$ & $\begin{array}{c}\text { GSK269962A } \\
1 \mathrm{uM} \\
\end{array}$ & $\begin{array}{l}\text { sunitinit } \\
0.1 \mathrm{uM}\end{array}$ & $1 \mathrm{uM}$ \\
\hline & LRRK2 wild type & $72 \pm 11$ & $77 \pm 13$ & $20 \pm 3$ & $22 \pm 2$ & $98 \pm 22$ & $71 \pm 0$ & $42 \pm 2$ & $9 \pm 0$ \\
\hline & LRRK2 G2019S & $26 \pm 1$ & $67 \pm 3$ & $\underline{13} \pm 1$ & $\underline{11} \pm 1$ & $97 \pm 5$ & $66 \pm 1$ & $\underline{14} \pm 1$ & $\underline{\mathbf{3}} \pm 0$ \\
\hline & ROCK 2 & $\underline{7} \pm 2$ & $\underline{3} \pm 1$ & $\underline{3} \pm 0$ & $\underline{4} \pm 1$ & $\underline{3} \pm 1$ & $1 \pm 1$ & $92 \pm 2$ & $67 \pm 5$ \\
\hline & MKK1 & $6 \overline{3} \pm 3$ & $4 \overline{1} \pm 2$ & $6 \overline{9} \pm 15$ & $7 \overline{1} \pm 17$ & $6 \overline{1} \pm 13$ & $5 \overline{4} \pm 2$ & $37 \pm 2$ & $\underline{16} \pm 1$ \\
\hline & ERK1 & $109 \pm 6$ & $104 \pm 2$ & $105 \pm 8$ & $92 \pm 1$ & $90 \pm 1$ & $106 \pm 2$ & $108 \pm 2$ & $104 \pm 7$ \\
\hline & ERK2 & $102 \pm 8$ & $98 \pm 2$ & $94 \pm 4$ & $94 \pm 2$ & $94 \pm 9$ & $105 \pm 4$ & $101 \pm 1$ & $103 \pm 10$ \\
\hline & JNK1 & $105 \pm 9$ & $115 \pm 8$ & $96 \pm 5$ & $106 \pm 6$ & $111 \pm 3$ & $105 \pm 9$ & $123 \pm 16$ & $104 \pm 5$ \\
\hline & JNK2 & $90 \pm 5$ & $94 \pm 4$ & $91 \pm 6$ & $96 \pm 9$ & $101 \pm 0$ & $101 \pm 12$ & $101 \pm 8$ & $88 \pm 1$ \\
\hline & p38a MAPK & $98 \pm 1$ & $108 \pm 13$ & $109 \pm 11$ & $103 \pm 4$ & $103 \pm 6$ & $108 \pm 6$ & $111 \pm 2$ & $103 \pm 0$ \\
\hline & p38b MAPK & $90 \pm 6$ & $111 \pm 2$ & $107 \pm 2$ & $101 \pm 10$ & $97 \pm 8$ & $113 \pm 2$ & $116 \pm 0$ & $113 \pm 4$ \\
\hline & p38g MAPK & $97 \pm 9$ & $99 \pm 5$ & $101 \pm 18$ & $96 \pm 13$ & $112 \pm 13$ & $72 \pm 14$ & $109 \pm 21$ & $98 \pm 18$ \\
\hline & p38d MAPK & $100 \pm 4$ & $77 \pm 1$ & $93 \pm 4$ & $86 \pm 1$ & $94 \pm 7$ & $86 \pm 7$ & $110 \pm 2$ & $107 \pm 1$ \\
\hline & ERK8 & $76 \pm 1$ & $68 \pm 44$ & $91 \pm 11$ & $83 \pm 6$ & $95 \pm 0$ & $27 \pm 1$ & $85 \pm 1$ & $59 \pm 4$ \\
\hline & RSK1 & $\underline{11} \pm 15$ & $14 \pm 5$ & $57 \pm 1$ & $26 \pm 3$ & $20 \pm 25$ & $12 \pm 1$ & $38 \pm 53$ & $78 \pm 9$ \\
\hline & RSK2 & $\overline{41} \pm 6$ & $\overline{31} \pm 1$ & $76 \pm 1$ & $42 \pm 5$ & $48 \pm 14$ & $\overline{24} \pm 4$ & $60 \pm 23$ & $41 \pm 5$ \\
\hline & PDK1 & $112 \pm 4$ & $97 \pm 1$ & $74 \pm 5$ & $120 \pm 9$ & $120 \pm 5$ & $117 \pm 0$ & $124 \pm 2$ & $95 \pm 1$ \\
\hline & PKBa & $27 \pm 7$ & $27 \pm 1$ & $64 \pm 8$ & $56 \pm 14$ & $72 \pm 16$ & $26 \pm 0$ & $87 \pm 10$ & $73 \pm 6$ \\
\hline & $\mathrm{PKBb}$ & $100 \pm 3$ & $77 \pm 11$ & $112 \pm 9$ & $105 \pm 1$ & $104 \pm 8$ & $64 \pm 1$ & $101 \pm 4$ & $88 \pm 2$ \\
\hline & SGK1 & $55 \pm 13$ & $69 \pm 21$ & $92 \pm 8$ & $50 \pm 8$ & $131 \pm 0$ & $24 \pm 9$ & $79 \pm 26$ & $26 \pm 5$ \\
\hline & S6K1 & $\underline{11} \pm 1$ & $\underline{13} \pm 0$ & $72 \pm 2$ & $70 \pm 11$ & $24 \pm 4$ & $\underline{\mathbf{2}} \pm 0$ & $82 \ddagger 4$ & $41 \pm 3$ \\
\hline & PKA & $\overline{89} \pm 10$ & $\overline{21} \pm 0$ & $76 \pm 5$ & $106 \pm 16$ & $88 \pm 24$ & $2 \overline{2} \pm 3$ & $104 \pm 3$ & $97 \pm 3$ \\
\hline & PRK2 & $\underline{12} \pm 0$ & $\underline{6} \pm 0$ & $70 \pm 69$ & $\underline{7} \pm 3$ & $40 \pm 3$ & $\underline{\mathbf{2}} \pm 0$ & $98 \pm 3$ & $81 \pm 17$ \\
\hline & PKCa & $\overline{64} \pm 5$ & $3 \overline{9} \pm 5$ & $70 \pm 11$ & $5 \overline{2} \pm 1$ & $82 \pm 4$ & $3 \overline{2} \pm 0$ & $93 \pm 13$ & $77 \pm 9$ \\
\hline & $\mathrm{PKCz}$ & $94 \pm 8$ & $66 \pm 2$ & $86 \pm 6$ & $69 \pm 2$ & $81 \pm 4$ & $\underline{5} \pm 1$ & $110 \pm 1$ & $94 \pm 6$ \\
\hline & PKD1 & $60 \pm 3$ & $25 \pm 1$ & $79 \pm 16$ & $89 \pm 0$ & $85 \pm 3$ & $8 \overline{5} \pm 6$ & $79 \pm 7$ & $34 \pm 5$ \\
\hline & MSK1 & $\underline{17} \pm 0$ & $\underline{14} \pm 1$ & $39 \pm 4$ & $41 \pm 8$ & $\underline{20} \pm 1$ & $1 \pm 0$ & $94 \pm 12$ & $60 \pm 8$ \\
\hline & MNK1 & $\overline{76} \pm 8$ & $\underline{20} \pm 3$ & $50 \pm 6$ & $\underline{\mathbf{2 0}} \pm 10$ & $\overline{91} \pm 6$ & $86 \pm 2$ & $99 \pm 10$ & $83 \pm 5$ \\
\hline & MNK2 & $77 \pm 4$ & $\overline{27} \pm 6$ & $49 \pm 9$ & $\overline{44} \pm 2$ & $88 \pm 6$ & $51 \pm 2$ & $83 \pm 17$ & $80 \pm 7$ \\
\hline 10 & MAPKAP-K2 & $96 \pm 9$ & $91 \pm 9$ & $93 \pm 0$ & $85 \pm 4$ & $91 \pm 1$ & $88 \pm 1$ & $91 \pm 17$ & $95 \pm 5$ \\
\hline m & PRAK & $88 \pm 16$ & $93 \pm 12$ & $87 \pm 17$ & $88 \pm 14$ & $92 \pm 17$ & $86 \pm 13$ & $102 \pm 1$ & $75 \pm 15$ \\
\hline$\simeq$ & CAMKKb & $93 \pm 3$ & $95 \pm 5$ & $81 \pm 6$ & $102 \pm 8$ & $100 \pm 1$ & $98 \pm 7$ & $87 \pm 12$ & $39 \pm 6$ \\
\hline ఠ & CAMK1 & $108 \pm 14$ & $109 \pm 4$ & $105 \pm 2$ & $85 \pm 22$ & $102 \pm 18$ & $122 \pm 31$ & $113 \pm 19$ & $66 \pm 2$ \\
\hline 8 & SmMLCK & $66 \pm 3$ & $66 \pm 6$ & $96 \pm 5$ & $70 \pm 1$ & $73 \pm 3$ & $65 \pm 3$ & $49 \pm 2$ & $26 \pm 3$ \\
\hline N & PHK & $89 \pm 4$ & $46 \pm 6$ & $26 \pm 2$ & $79 \pm 1$ & $97 \pm 8$ & $92 \pm 3$ & $\underline{11} \pm 1$ & $\underline{2} \pm 0$ \\
\hline m & CHK1 & $87 \pm 1$ & $103 \pm 5$ & $89 \pm 14$ & $96 \pm 9$ & $111 \pm 6$ & $88 \pm 2$ & $\overline{76} \pm 0$ & $3 \overline{3} \pm 3$ \\
\hline$\stackrel{m}{\infty}$ & CHK2 & $89 \pm 13$ & $34 \pm 2$ & $44 \pm 0$ & $93 \pm 7$ & $102 \pm 6$ & $52 \pm 1$ & $23 \pm 1$ & $\underline{\mathbf{5}} \pm 1$ \\
\hline N & GSK3b & $91 \pm 11$ & $91 \pm 5$ & $72 \pm 28$ & $92 \pm 3$ & $93 \pm 13$ & $12 \pm 0$ & $108 \pm 3$ & $9 \overline{3} \pm 11$ \\
\hline オ & CDK2-Cyclin A & $98 \pm 5$ & $82 \pm 6$ & $74 \pm 7$ & $72 \pm 4$ & $49 \pm 34$ & $\overline{50} \pm 3$ & $98 \pm 2$ & $87 \pm 5$ \\
\hline$\simeq$ & PLK1 & $101 \pm 9$ & $111 \pm 14$ & $105 \pm 1$ & $108 \pm 16$ & $102 \pm 5$ & $105 \pm 6$ & $103 \pm 9$ & $109 \pm 10$ \\
\hline $0^{\circ}$ & Aurora B & $59 \pm 1$ & $51 \pm 1$ & $\underline{11} \pm 2$ & $79 \pm 1$ & $59 \pm 5$ & $36 \pm 0$ & $72 \pm 5$ & $29 \pm 4$ \\
\hline$\div$ & AMPK & $72 \pm 2$ & $60 \pm 1$ & $\overline{45} \pm 4$ & $77 \pm 4$ & $78 \pm 15$ & $87 \pm 0$ & $51 \pm 3$ & $\underline{15} \pm 4$ \\
\hline$\ddot{0}$ & MARK3 & $84 \pm 2$ & $79 \pm 2$ & $56 \pm 3$ & $105 \pm 5$ & $98 \pm 7$ & $67 \pm 0$ & $77 \pm 4$ & $\overline{36} \pm 4$ \\
\hline 응 & BRSK2 & $65 \pm 3$ & $38 \pm 2$ & $\frac{17}{70} \pm 4$ & $43 \pm 4$ & $91 \pm 10$ & $95 \pm 7$ & $94 \pm 0$ & $40 \pm 6$ \\
\hline 0 & MELK & $76 \pm 2$ & $\underline{19} \pm 1$ & $\overline{70} \pm 4$ & $79 \pm 2$ & $83 \pm 0$ & $44 \pm 3$ & $55 \pm 6$ & $\underline{17} \pm 1$ \\
\hline$\Phi$ & CK1 & $100 \pm 0$ & $77 \pm 1$ & $112 \pm 9$ & $107 \pm 12$ & $106 \pm 3$ & $102 \pm 2$ & $50 \pm 2$ & $\underline{10} \pm 0$ \\
\hline$\omega$ & CK2 & $84 \pm 4$ & $81 \pm 9$ & $84 \pm 17$ & $85 \pm 2$ & $82 \pm 0$ & $88 \pm 2$ & $91 \pm 2$ & $\overline{72} \pm 1$ \\
\hline 1 & DYRK1A & $96 \pm 16$ & $102 \pm 3$ & $96 \pm 6$ & $96 \pm 3$ & $98 \pm 7$ & $95 \pm 7$ & $103 \pm 6$ & $83 \pm 8$ \\
\hline ح & DYRK2 & $89 \pm 1$ & $80 \pm 2$ & $84 \pm 2$ & $92 \pm 5$ & $92 \pm 7$ & $64 \pm 2$ & $92 \pm 6$ & $76 \pm 9$ \\
\hline ભ & DYRK3 & $99 \pm 2$ & $39 \pm 5$ & $94 \pm 0$ & $100 \pm 6$ & $94 \pm 6$ & $77 \pm 9$ & $104 \pm 5$ & $84 \pm 3$ \\
\hline 0 & NEK2a & $103 \pm 2$ & $98 \pm 9$ & $99 \pm 3$ & $104 \pm 0$ & $106 \pm 5$ & $94 \pm 5$ & $103 \pm 12$ & $87 \pm 6$ \\
\hline 0 & NEK6 & $96 \pm 8$ & $107 \pm 28$ & $99 \pm 16$ & $80 \pm 18$ & $87 \pm 24$ & $82 \pm 19$ & $95 \pm 26$ & $83 \pm 15$ \\
\hline Ш & $\mathrm{IKKb}$ & $86 \pm 6$ & $79 \pm 4$ & $100 \pm 8$ & $79 \pm 4$ & $97 \pm 7$ & $83 \pm 3$ & $90 \pm 9$ & $80 \pm 4$ \\
\hline Ш & PIM1 & $90 \pm 14$ & $84 \pm 14$ & $90 \pm 5$ & $94 \pm 12$ & $89 \pm 8$ & $31 \pm 6$ & $95 \pm 5$ & $86 \pm 14$ \\
\hline 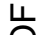 & PIM2 & $104 \pm 1$ & $111 \pm 3$ & $91 \pm 7$ & $102 \pm 3$ & $106 \pm 9$ & $89 \pm 5$ & $111 \pm 7$ & $104 \pm 2$ \\
\hline 0 & PIM3 & $75 \pm 1$ & $81 \pm 1$ & $96 \pm 1$ & $87 \pm 2$ & $89 \pm 1$ & $35 \pm 4$ & $84 \pm 1$ & $50 \pm 8$ \\
\hline$z$ & SRPK1 & $90 \pm 12$ & $92 \pm 10$ & $125 \pm 1$ & $65 \pm 35$ & $71 \pm 8$ & $90 \pm 13$ & $80 \pm 0$ & $86 \pm 4$ \\
\hline$\overline{0}$ & MST2 & $44 \pm 4$ & $51 \pm 2$ & $41 \pm 2$ & $61 \pm 8$ & $108 \pm 3$ & $98 \pm 2$ & $59 \pm 7$ & $\underline{16} \pm 2$ \\
\hline & $\mathrm{EF} 2 \mathrm{~K}$ & $103 \pm 14$ & $120 \pm 4$ & $99 \pm 24$ & $81 \pm 2$ & $99 \pm 4$ & $113 \pm 30$ & $97 \pm 15$ & $1 \overline{06} \pm 26$ \\
\hline תִ & HIPK2 & $109 \pm 3$ & $127 \pm 6$ & $100 \pm 0$ & $104 \pm 1$ & $104 \pm 3$ & $\underline{17} \pm 0$ & $79 \pm 2$ & $30 \pm 1$ \\
\hline 는 & PAK4 & $104 \pm 4$ & $99 \pm 17$ & $99 \pm 7$ & $101 \pm 23$ & $95 \pm 1$ & $\overline{95} \pm 3$ & $99 \pm 9$ & $72 \pm 5$ \\
\hline Ш & PAK5 & $113 \pm 7$ & $112 \pm 16$ & $100 \pm 1$ & $101 \pm 11$ & $107 \pm 5$ & $102 \pm 4$ & $121 \pm 4$ & $99 \pm 3$ \\
\hline H & PAK6 & $103 \pm 5$ & $110 \pm 8$ & $103 \pm 7$ & $96 \pm 7$ & $109 \pm 7$ & $106 \pm 2$ & $115 \pm 8$ & $102 \pm 10$ \\
\hline Ш & MST4 & $82 \pm 2$ & $79 \pm 8$ & $90 \pm 7$ & $67 \pm 7$ & $70 \pm 5$ & $86 \pm 9$ & $87 \pm 5$ & $80 \pm 6$ \\
\hline$\perp$ & TBK1 & $98 \pm 5$ & $110 \pm 3$ & $98 \pm 7$ & $69 \pm 9$ & $101 \pm 0$ & $77 \pm 2$ & $106 \pm 9$ & $54 \pm 1$ \\
\hline Г & IKKe & $99 \pm 2$ & $95 \pm 8$ & $80 \pm 4$ & $76 \pm 8$ & $109 \pm 15$ & $66 \pm 21$ & $101 \pm 1$ & $63 \pm 3$ \\
\hline $\bar{\Omega}$ & GCK & $73 \pm 7$ & $55 \pm 4$ & $56 \pm 1$ & $89 \pm 4$ & $97 \pm 7$ & $78 \pm 9$ & $45 \pm 2$ & $\underline{11} \pm 1$ \\
\hline J & IRAK4 & $87 \pm 4$ & $69 \pm 7$ & $102 \pm 7$ & $101 \pm 8$ & $94 \pm 1$ & $78 \pm 2$ & $76 \pm 19$ & $\overline{23} \pm 4$ \\
\hline$<$ & NUAK1 & $36 \pm 11$ & $21 \pm 2$ & $51 \pm 10$ & $106 \pm 1$ & $51 \pm 1$ & $39 \pm 4$ & $30 \pm 1$ & $\underline{15} \pm 3$ \\
\hline$\underline{\Theta}$ & MLK1 & $75 \pm 16$ & $56 \pm 5$ & $82 \pm 18$ & $90 \pm 19$ & $81 \pm 14$ & $77 \pm 7$ & $74 \pm 2$ & $\overline{40} \pm 0$ \\
\hline$c \Omega$ & MINK1 & $68 \pm 21$ & $54 \pm 6$ & $104 \pm 5$ & $81 \pm 0$ & $75 \pm 9$ & $70 \pm 4$ & $46 \pm 1$ & $8 \pm 0$ \\
\hline 可 & MLK3 & $45 \pm 60$ & $72 \pm 8$ & $85 \pm 9$ & $110 \pm 3$ & $116 \pm 23$ & $97 \pm 1$ & $72 \pm 9$ & $2 \overline{6} \pm 1$ \\
\hline I & LKB1 & $50 \pm 0$ & $33 \pm 0$ & $50 \pm 3$ & $52 \pm 12$ & $58 \pm 1$ & $58 \pm 4$ & $59 \pm 1$ & $52 \pm 2$ \\
\hline$\vdash$ & HER4 & $97 \pm 5$ & $129 \pm 4$ & $107 \pm 2$ & $69 \pm 9$ & $50 \pm 2$ & $93 \pm 3$ & $126 \pm 14$ & $103 \pm 9$ \\
\hline & TTK & $82 \pm 2$ & $73 \pm 5$ & $93 \pm 9$ & $73 \pm 2$ & $83 \pm 0$ & $69 \pm 0$ & $99 \pm 5$ & $72 \pm 6$ \\
\hline & Src & $111 \pm 2$ & $120 \pm 6$ & $81 \pm 0$ & $90 \pm 4$ & $85 \pm 4$ & $105 \pm 0$ & $98 \pm 7$ & $52 \pm 1$ \\
\hline & Lck & $79 \pm 29$ & $109 \pm 7$ & $74 \pm 1$ & $83 \pm 32$ & $82 \pm 7$ & $102 \pm 4$ & $51 \pm 4$ & $\underline{11} \pm 2$ \\
\hline & CSK & $95 \pm 7$ & $104 \pm 12$ & $101 \pm 4$ & $104 \pm 14$ & $106 \pm 19$ & $98 \pm 15$ & $98 \pm 10$ & $\overline{89} \pm 2$ \\
\hline & FGF-R1 & $83 \pm 2$ & $65 \pm 5$ & $35 \pm 3$ & $95 \pm 9$ & $95 \pm 3$ & $96 \pm 18$ & $77 \pm 2$ & $29 \pm 0$ \\
\hline & IRR & $89 \pm 1$ & $86 \pm 4$ & $88 \pm 2$ & $91 \pm 1$ & $106 \pm 1$ & $61 \pm 7$ & $90 \pm 1$ & $77 \pm 24$ \\
\hline & EPH A2 & $95 \pm 8$ & $94 \pm 6$ & $49 \pm 5$ & $106 \pm 4$ & $105 \pm 12$ & $108 \pm 4$ & $104 \pm 3$ & $91 \pm 9$ \\
\hline & SYK & $112 \pm 11$ & $104 \pm 1$ & $104 \pm 2$ & $94 \pm 8$ & $78 \pm 9$ & $73 \pm 5$ & $107 \pm 13$ & $94 \pm 10$ \\
\hline & YES1 & $111 \pm 4$ & $136 \pm 6$ & $76 \pm 8$ & $96 \pm 5$ & $58 \pm 5$ & $114 \pm 11$ & $30 \pm 3$ & $\underline{7} \pm 1$ \\
\hline & IGF-1R & $91 \pm 9$ & $92 \pm 1$ & $113 \pm 2$ & $99 \pm 5$ & $97 \pm 1$ & $104 \pm 7$ & $84 \pm 1$ & $3 \overline{3} \pm 6$ \\
\hline & VEG-FR & $115 \pm 1$ & $70 \pm 4$ & $82 \pm 20$ & $105 \pm 4$ & $117 \pm 12$ & $91 \pm 7$ & $33 \pm 0$ & $\underline{9} \pm 0$ \\
\hline & BTK & $108 \pm 20$ & $112 \pm 21$ & $82 \pm 9$ & $85 \pm 17$ & $66 \pm 3$ & $107 \pm 4$ & $90 \pm 1$ & $5 \overline{2} \pm 12$ \\
\hline & IR-HIS & $100 \pm 4$ & $105 \pm 1$ & $108 \pm 6$ & $113 \pm 1$ & $107 \pm 13$ & $93 \pm 1$ & $104 \pm 7$ & $65 \pm 5$ \\
\hline & EPH-B3 & $111 \pm 2$ & $65 \pm 9$ & $69 \pm 11$ & $83 \pm 12$ & $87 \pm 9$ & $92 \pm 10$ & $107 \pm 9$ & $103 \pm 0$ \\
\hline
\end{tabular}

